\title{
Women Veterans' Descriptions of the Patient-Provider Interaction with Civilian Providers
}

\author{
Billie S. Vance \\ billie.vance@hsc.wvu.edu
}

Follow this and additional works at: https://researchrepository.wvu.edu/etd

Part of the Family Practice Nursing Commons, and the Other Nursing Commons

\section{Recommended Citation}

Vance, Billie S., "Women Veterans' Descriptions of the Patient-Provider Interaction with Civilian Providers" (2020). Graduate Theses, Dissertations, and Problem Reports. 7618.

https://researchrepository.wvu.edu/etd/7618

This Dissertation is protected by copyright and/or related rights. It has been brought to you by the The Research Repository @WVU with permission from the rights-holder(s). You are free to use this Dissertation in any way that is permitted by the copyright and related rights legislation that applies to your use. For other uses you must obtain permission from the rights-holder(s) directly, unless additional rights are indicated by a Creative Commons license in the record and/ or on the work itself. This Dissertation has been accepted for inclusion in WVU Graduate Theses, Dissertations, and Problem Reports collection by an authorized administrator of The Research Repository @ WVU.

For more information, please contact researchrepository@mail.wvu.edu. 
Women Veterans' Descriptions of the Patient-Provider Interaction with Civilian Providers

Billie S. Vance

Dissertation submitted to the School of Nursing at West Virginia University in partial fulfillment of the requirements for the degree of

Doctor of Philosophy in

Nursing

Kari Sand-Jecklin, EdD, MSN, RN, AHN-BC, Chair

Roger Carpenter, $\mathrm{PhD}, \mathrm{RN}, \mathrm{NE}-\mathrm{BC}, \mathrm{CNE}$

Suzy Mascaro Walter, PhD, MSN, APRN, FNP-BC, CNRN

Todd Fredricks, D.O.

Department of Adult Health

Morgantown, West Virginia

2020

Key words: Women Veterans; Interaction Model of Client Health Behavior; Patient-Provider Interaction; Civilian Providers

Copyright 2020 Billie S. Vance 


\begin{abstract}
Women Veterans' Descriptions of the Patient-Provider Interaction with Civilian Providers
\end{abstract}

Billie S. Vance

BACKGROUND: Women veterans require care for unique gender, and war-related health issues. Concerns exist regarding non-Veterans Health Administration (VHA), civilian healthcare providers' preparedness to deliver care to the veteran population. Health outcomes are influenced by the quality of care provided to patients. There is a significant gap in the literature with regard to woman veterans' perspectives about the quality of the patient-provider interaction between women veterans and non-VHA, civilian providers.

PURPOSE: The purpose of this study was to describe women veterans' perspectives about interactions between themselves and civilian providers and to critically analyze the veterans' data to further inform the use of the Interaction Model of Client Health Behavior.

METHODS: A qualitative descriptive methodology was employed. A purposive sampling plan was used to recruit 13 women veteran participants who exclusively obtained care from nonVHA, civilian healthcare providers. Data collection and analysis was guided by directed content analysis.

RESULTS: Six themes surrounding the four factors that comprise the patient-provider interaction element of the model emerged and were coherent with the model. Affective support is knowing me as a person by hearing my story and being attentive to my needs. Professionaltechnical competence is recognizing and acknowledging women are veterans, comprehending the military experience, and being thorough and accepting accountability for attending to a health concern. Information giving is uncomplicated explanation that facilitates decision making. Decisional control is collaborating by seeking input, providing options, and supporting decisions.

CONCLUSIONS: The themes indicate that among non-VHA, civilian providers, there is an absence of consistent screening for military service in female patients, a deficit in knowledge regarding the role and experiences of women during military service, and the need for additional training about military-related health conditions. Further, the themes highlight the importance of the woman veteran's story, as well as the need to recognize and acknowledge the service of women veterans. Finally, the themes inform the use of the IMCHB to guide research, practice, and policy related to the care of women veterans who obtain care from non-VHA, civilian providers. 


\section{Acknowledgements}

I would like to thank the many people who contributed to this work, starting with my dissertation committee: Dr. Kari Sand-Jecklin, whose guidance, support, and words of encouragement (and her willingness to answer phone calls on the weekend and after hours) kept me focused and moving forward; Dr. Roger Carpenter for his guidance and mentorship during analysis and for sharing his expertise in qualitative descriptive methodology and directed content analysis; Dr. Suzy Walter for her willingness to be part of my committee, words of encouragement, and for providing critique of my work ; Dr. Todd Fredricks whose commitment to improving the care of those who have served, as well as his personal military service, are an

inspiration - thank you for your service. To my original PhD cohort who finished before me and the cohort that adopted me, thank you for laughing with me, crying with me, commiserating with me, (sometimes drinking with me), and pushing forward with me. I am thankful for my children, Clay and Josi, for being patient with me and loving me during the many days and nights I sat glued to the table, surrounded by papers, working on this degree. I am thankful for my husband, Jerry, whose love, friendship, and encouragement got me through the remainder of my $\mathrm{PhD}$ journey. Finally, for the rest of my family, friends, and colleagues who encouraged and supported me all along the way and pressured me to continue when I wanted to quit.

Research reported in this publication was supported by the National Institute of General Medical Sciences of the National Institutes of Health under Award Number 5U54GM104942-04. The content is solely the responsibility of the authors and does not necessarily represent the official views of the National Institutes of Health. 


\section{Dedication}

I dedicate this dissertation to women veterans. Their historical and continued service to our country is remarkable. Let no one ever doubt their commitment, service, or worth as a member of our military. I pray this small contribution will help to improve the quality of civilian delivered healthcare for women veterans. 


\section{Table of Contents}

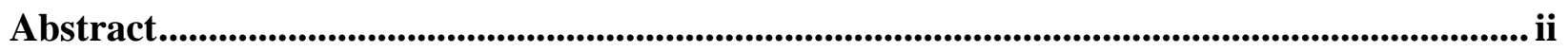

Acknowledgements ....................................................................................................................

Dedication ................................................................................................................................................... iv

Chapter 1: Introduction ..................................................................................................................................... 1

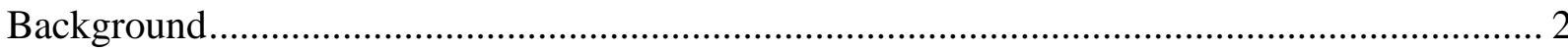

Theoretical Rationale

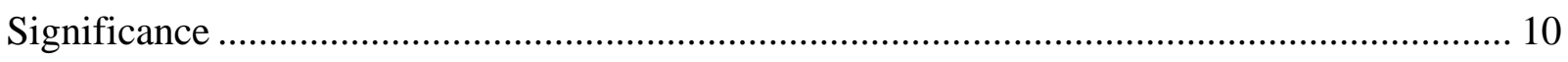

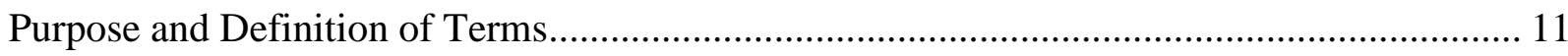

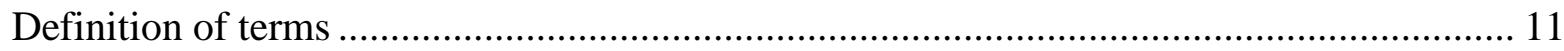

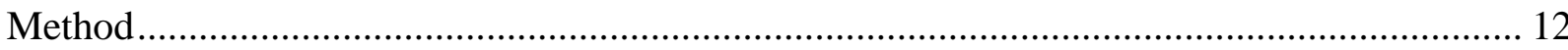

Chapter 2: Literature Review...................................................................................................... 13

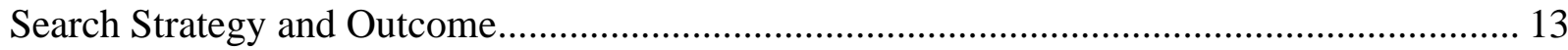

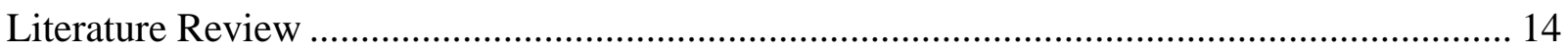

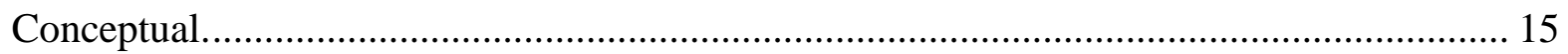

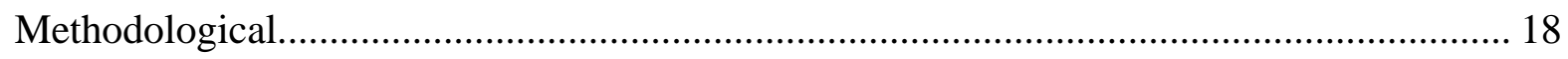

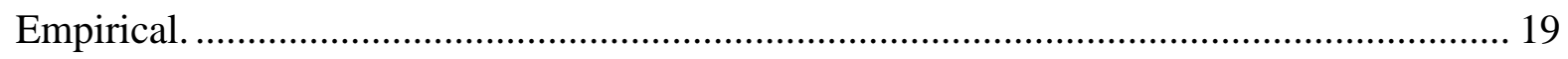

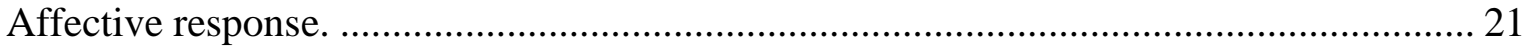

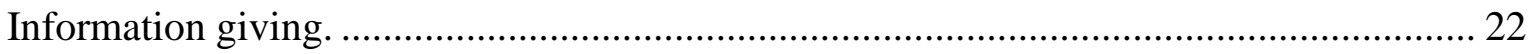

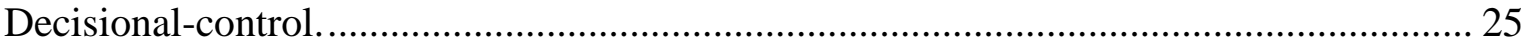

Professional-technical competence.............................................................................. 27

Findings outside of the patient-provider interaction factors............................................. 30

Continuity, time, and accessibility …………………………................................... 30

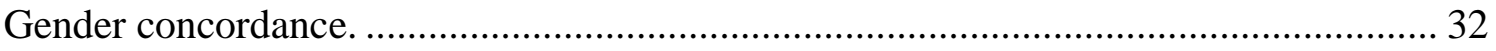

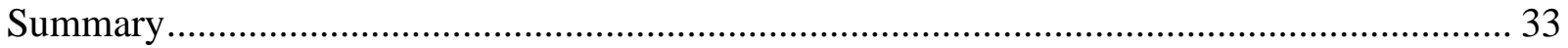

Chapter 3: Method.............................................................................................................................. 36

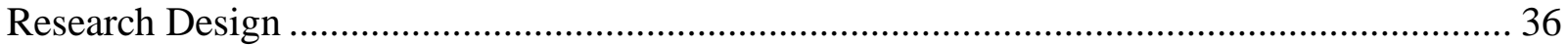

Protection of Human Rights and Ethical Considerations .......................................................... 37

Population and Sample Selection ……………………................................................... 38

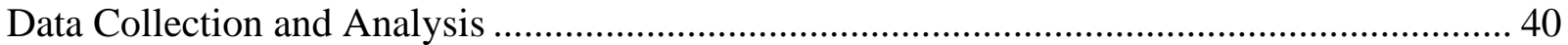

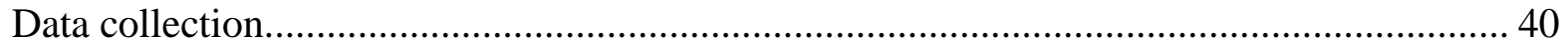

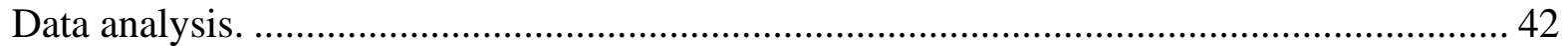

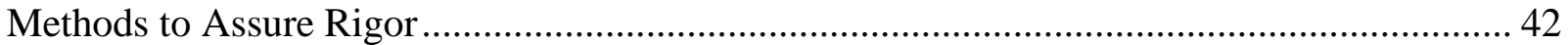

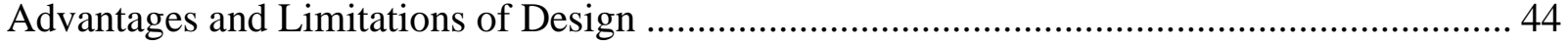




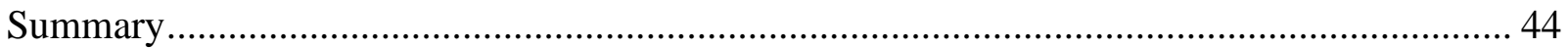

Chapter 4: Results....................................................................................................................... 45

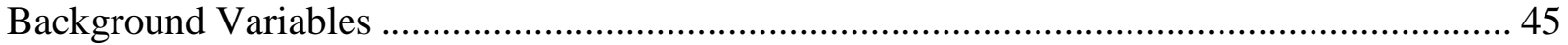

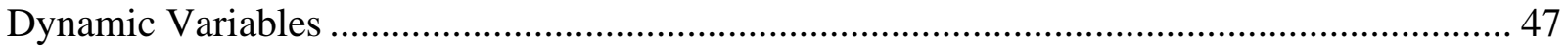

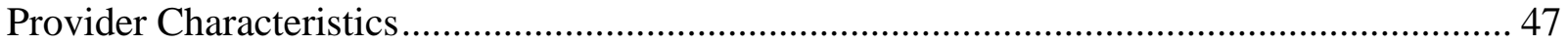

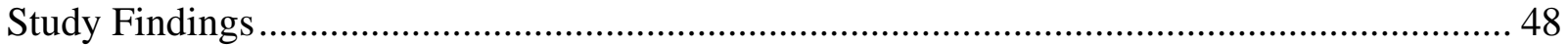

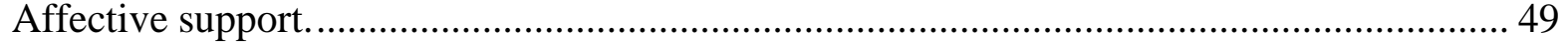

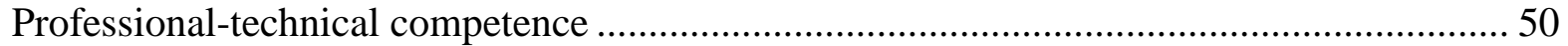

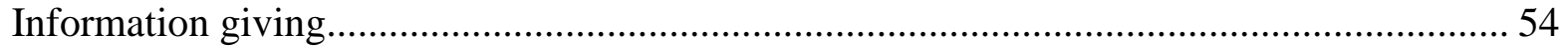

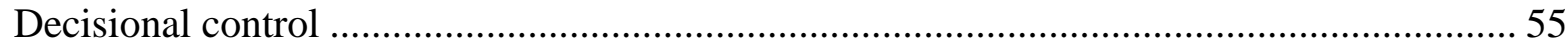

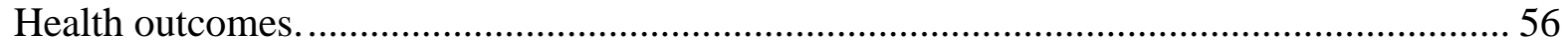

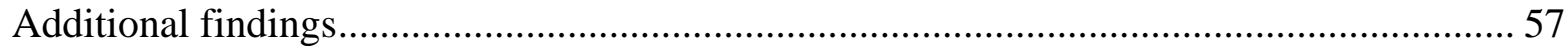

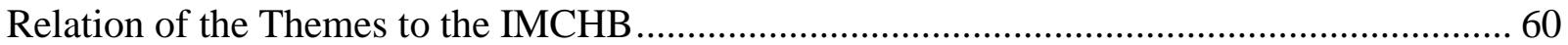

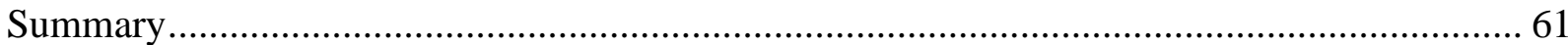

Chapter 5: Conclusions .................................................................................................................... 62

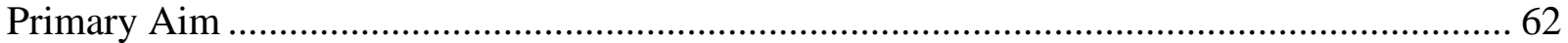

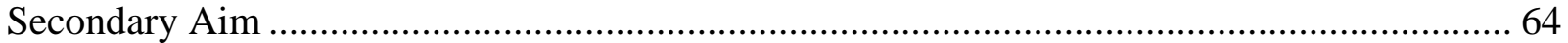

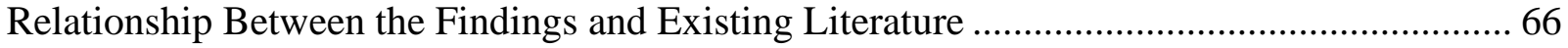

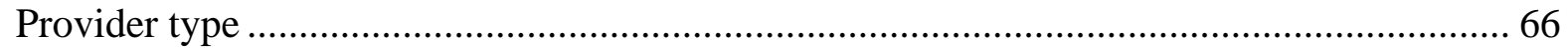

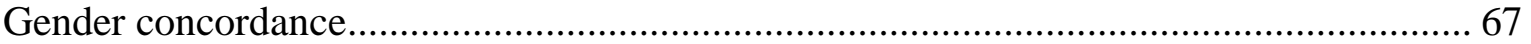

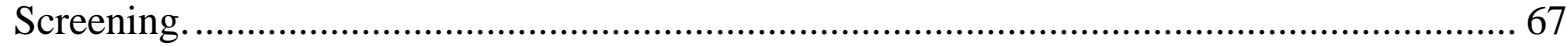

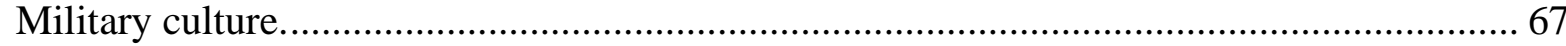

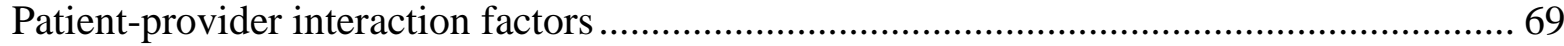

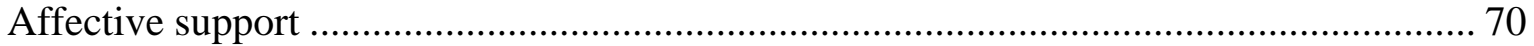

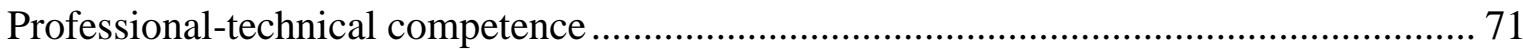

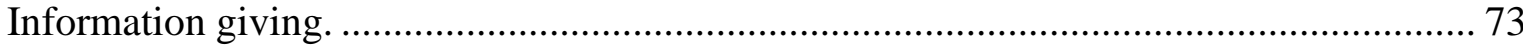

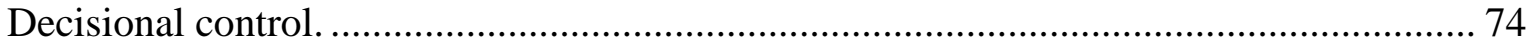

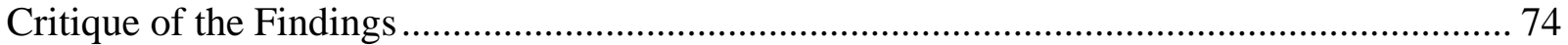

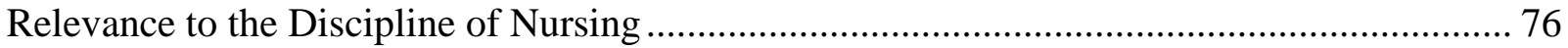

Implications for Policy, Research, and Practice .................................................................... 78

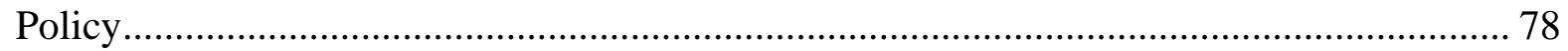

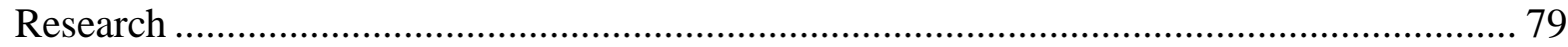

Recruitment

Practice. 


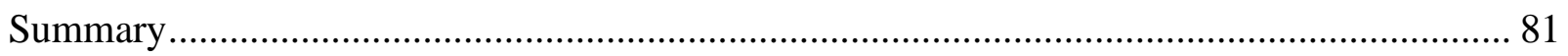

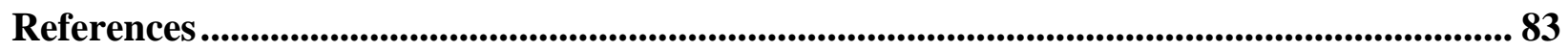

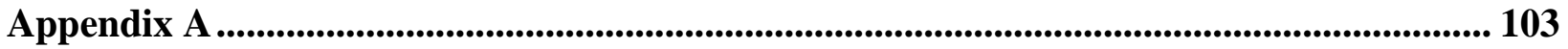

Figure 1. Interaction model of client health behavior ............................................ 103

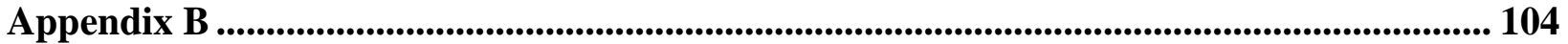

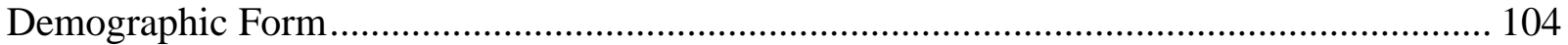

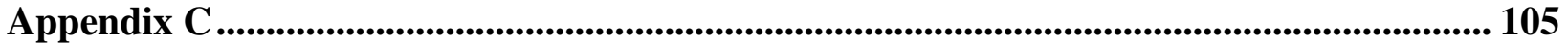

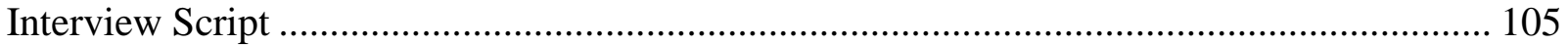

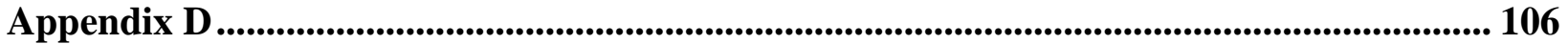

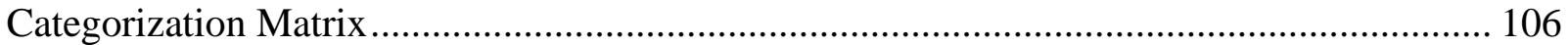

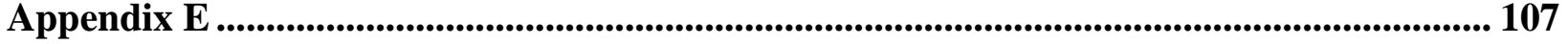

Figure 2. Directed content analysis method ........................................................ 107

Appendix F ....................................................................................................................................... 108

Table 1. Themes and Associated Condensed Meaning Units ...................................... 108 


\section{Chapter 1: Introduction}

In the United States (US) there are currently more than two million female veterans, and this number is expected to increase annually by approximately 18,000 (National Center for Veterans Analysis and Statistics [NCVAS], 2017). Approximately 33\% of the contemporary female veteran population served during the Gulf War 2 or Post-9/11 era (September 2001 to present) (NCVAS, 2016), with women representing almost $12 \%$ of the military persons deployed during the Post-9/11 conflicts (DAV, 2018). It is well established that women veterans returning from war require care for unique gender, and war-related health issues (Creech et al., 2019). While the Veterans Health Administration (VHA) has made significant efforts to transform the VHA healthcare system to provide more inclusive, sensitive, and gender appropriate care for women veterans (Maisel et al., 2015), only a minority of women veterans obtain care at from VHA healthcare facilities (Washington, Farmer, Mor, Canning, \& Yano, 2015). The remaining portion are seeking care from within the non-VHA, civilian healthcare sector.

Despite the recognition of the need for gender-specific research related to women veterans, only about $2 \%$ of the veteran literature mentions women veterans, and even fewer publications focus solely on women veterans as the primary population of study (Dodds \& Kiernan, 2019). In addition, the majority of research on women veterans is dominated by studies about women veterans who are accessing VHA services, with little examination of women veterans accessing healthcare outside of the VHA (Danan et al, 2017). Further, even fewer studies investigate the healthcare experience of women veterans or their narratives (Dodds \& Kiernan, 2019). As a result, there is a significant gap in the literature about woman veterans' who obtain care from the non-VHA, civilian healthcare sector. Thus, the problem that this 
investigation addressed is that little is known about the women veterans' experiences with nonVHA, civilian healthcare providers.

\section{Background}

Enlistment of women in the military increased post 9/11, during the Operations Iraqi Freedom (OIF), Enduring Freedom (OEF), and New Dawn (OND) (Atkins, 2013). Subsequently, women veterans are one of the fastest growing segments of the United States (U.S) veteran population (NCVAS, 2017). To date, approximately $9.8 \%$ of all veterans are women (NCVAS, 2017).

Compared to previous wars, women serving in OIF, OEF, and OND were often subjected to multiple and longer deployments and served in or around combat theaters (Institute of Medicine $[\mathrm{IOM}], 2013)$. Thus, many women veterans return from war with deployment and/or combat related physical and psychological health issues which require ongoing healthcare (Batuman et al., 2011; Carlson, Stromwall, \& Lietz, 2013; Creech et al., 2019; Conrad \& Armstrong, 2016; IOM, 2013; Rivera \& Johnson, 2014). Coined the "invisible wounds" (often invisible to the eye and unrecognized or unacknowledged) of Iraq and Afghanistan, common health issues include: traumatic brain injury (TBI), depression, military sexual trauma (MST), substance use disorder (SUD), and post-traumatic stress disorder (PTSD) (Tanielian et al., 2008). During the transition from soldier to veteran, a decrease in both preventative health care services use, and overall physical and mental health has been observed among the military population (Villagran, Ledford, \& Canzona, 2015). Barriers to receiving health care exist for women veterans including the inability to take time off from work for healthcare visits, caregiver responsibilities, and transportation difficulties (Cordasco, Mengeling, Yano, \& Washington, 2016). Additionally, limited healthcare access, stigma of seeking care, availability of gender- 
specific care (Washington, Bean-Mayberry, Riopell, \& Yano, 2011) and affordability (Lahavot, Der-Martirosian, Simpson, \& Sadler, 2013) have been identified as barriers. Finally, women veterans often report a lack of knowledge about Veteran Health Administration (VHA) eligibility and services (U.S. Department of Veterans Affairs, 2015; Washington et al., 2015) as a reason for reduced healthcare service use.

Health risks and needs of women veterans are clinically complex (Creech et al., 2019) and uniquely different from that of male veterans (Boyd, Bradshaw, \& Robinson, 2013; Levander \& Overland, 2015; Katon \& Reiver, G., 2013; Resnick, Mallampalli, \& Carter, 2012). Compared to their male peers, women veterans experience higher rates of adverse mental health (Frayne et al., 2014; Hoglund \& Schwartz, 2014) and higher rates of physical and functional impairments (Runnals et al., 2014). Further, women veterans may experience gender specific health difficulties such as gynecological, reproductive, and urological symptomology, which may be worsened when comorbid with MST and/or PTSD (Maguen et al., 2012; Rivera \& Johnson, 2014). Women veterans with a history of MST are 1.26 times more likely to experience chronic pain conditions like irritable bowel syndrome, fibromyalgia, and chronic joint pain (Rogers et al., 2017), and a 1\% higher risk of obesity, than those without MST history (Pandey, Ashfaq, Dautirive, MacCarthy, \& Copeland, 2018).

The costs and sequelae of women veterans' health issues include increased morbidity and mortality from other medical illnesses, decreased work productivity, interpersonal and family dysfunction, homelessness, and an increasing rate of suicide (Carlson et al., 2013). Compared to their civilian counterparts, women veterans were more likely to experience poorer general health, higher incidence of depressive disorder (Lavahvot, Hoerster, Nelson, Simpson, \& Jakupcak, 2012), poorer functional status, and poorer mental health (Shen \& Sambamoorthi, 2012). 
Additionally, Hoffmire, Kemp, \& Bossarte (2015) reported that women veterans commit suicide at five to six times the rate of civilian women.

Veterans Health Administration health care facilities and providers offer evidence-based treatment for deployment/war associated health issues, such as PTSD, TBI, MST, and SUD (IOM, 2013). However, the preparation of VHA service personnel to provide gender specific and general care to women veterans has been under scrutiny (Bergman, Frankel, Hamilton, \& Yano, 2015). Responding to this concern, the VHA has made significant efforts to decrease barriers and to make comprehensive care for women veterans available at VHA health care centers, including the addition of designated women's health providers (Bastian et al., 2015; Mattocks, 2015; Meredith et al, 2017; Yano et al., 2011). However, not all women veterans receive care through the VHA.

Of the 1,965,534 separated Iraq/Afghanistan veterans, only $62 \%$ utilize VHA health care, of which women veterans account for only $12.3 \%$ (U.S. Department of Veterans Affairs, 2017). While most veterans are eligible to enroll for VHA health care, there are eligibility priorities imposed by annual aggregate funding appropriation; eligibility is determined by disability rating (Jackonis, Deyton, \& Hess, 2008). Without a service-connected disability, veterans are ineligible to receive VHA health care benefits. Thus, many women veterans receive care outside of the VHA setting within the civilian sector (Hinojosa, Hinojosa, Nelson, \& Nelson, 2010;

Washington et al., 2015). Other reasons women veterans may obtain civilian healthcare include: having insurance that covers healthcare outside of the VHA, more convenient location of the civilian health services, lack of awareness of eligibility for VHA services, and a feeling of not belonging in a healthcare system designed to deliver care to a male veteran majority (Bergman et al., 2015; Washington et al., 2015). In addition, VHA eligible veterans are seeking care within 
the civilian sector secondary to the Veterans' Access, Choice, and Accountability (Choice) Act of 2014 (U.S. Congress, 2014), which is now permanently replaced with the VHA Maintaining Systems and Strengthening Integrated Outside Networks (MISSION) Act of 2018 (Congressional Research Services [CRS], 2018). The impetus for the development and implementation of these acts was to expand access to healthcare services to veterans due to limitations in VHA services by enabling veterans to obtain care from community (non-VHA, civilian) settings and providers. Despite a statistically significant increase in access to care for women veterans since implementation of the Choice Act, Vance, Alhussain, \& Sambamoorthi (2019) found that there was no significant improvement in health-related quality of life outcomes (general, physical, and mental health, and functional status) for women veterans in 2017 compared to women veterans in 2013. This further highlights the need to understand more about the quality of care provided to women veterans.

Attention to the preparedness of civilian providers with regard to caring for veterans and their unique health issues is emerging in the literature. Civilian providers have reported only a moderate level of knowledge about military culture, military health risks, treatment for veterans' medical conditions, or referral and consultation services available from the VHA (Fredricks \& Nakazawa, 2015; Kilpatrick, Best, Smith, Kidler, \& Cornelison-Grant, 2011; Maiocco, Vance, \& Dichiacchio, in press). Additionally, many civilian providers report discomfort in discussing health related exposures and associated risks experienced by veterans while in the military or during deployment(s) (Fredricks \& Nakazawa, 2015; Koblinsky, Leslie, \& Cook, 2014; Maiocco, Stroupe, Snider, \& Vance, 2018; Maiocco et al., in press). Many civilian providers do not routinely screen their clients for a history of military service and/or deployment (Koblinsky et al., 2014; Tanielian et al., 2014). Civilian providers' poor understanding of the impact of 
veteran status on health and lack of knowledge about military culture may impede screening (Vest, Kulak, Hall, \& Homish, 2018a; Maiocco et al., in press). The latter is most concerning because help-seeking and/or self-disclosure of military service health issues by veterans may be negatively influenced by military ethos (or values). Military ethos promotes self-reliance and resilience, and indirectly discourages signs of weakness (Convoy \& Westphal, 2013; Tanielian et al., 2008; Weiss, Coll, \& Metal, 2011). Inquiring about a history of military service and experiences, as well as understanding military culture and ethos, are important in health screening of and care delivery to veterans (American Academy of Nursing [AAN], n.d; Meyer, 2015; Meyer, Writer, \& Brim, 2016).

With regard to the status of non-VHA, civilian delivered healthcare, there remains a little discussed component - the woman veteran's voice (Dodds \& Kiernan, 2019; Runnals et al., 2014; Wands, 2013). To date, only one study by Koblinsky, Schroeder, and Leslie (2017) explored women veterans' suggestions for improving both civilian and VHA delivered mental health care. Identified areas for improvement include the therapeutic relationship, clinical care environment, and health care system. The authors note that additional research is needed surrounding these areas for women veterans who do and do not use VHA health services. Systematic information regarding the experiences of veterans seeking care outside of the VHA health care systems is not available (Bagalman, 2013; Miller \& Intrator, 2012; U.S. Department of Veterans Affairs, 2017). Women veterans who exclusively use civilian health care are grossly underrepresented in the literature.

\section{Theoretical Rationale}

The interaction model of client health behavior (IMCHB) was developed by Cox (1982) to theoretically conceptualize the content and process of nursing care delivered to the client as an 
individual, and the subsequent outcomes related to that nursing care. The theory is the lens through which the interaction between the woman veteran patient and the civilian healthcare provider may be viewed. Major assumptions in the theory include: 1) clients are capable of and should be given the maximum amount of control in making choices about their health care behavior; 2) aspects of the client's singularity affect client choices; and 3) the role of the professional is that of teacher, counselor, and technician versus decision maker (p.46-47). The theory is comprised of three elements: client singularity, client-professional interaction, and health outcomes (Cox, 1982; 2003). To be consistent with current terminology, henceforth client will be referred to as patient, and professional as provider.

Patient singularity includes patients' (unique) background variables (demographic characteristics, social influence, previous health care experience, and environmental resources) and dynamic variables (motivation, cognitive appraisal, and affective response). Four factors that define the patient-provider interaction element include: the provision of health information (quantity, quality, and approach to delivery), affective support (attending to the patient's emotional arousal), decisional control (allowing an individual to participate in decision making), and professional-technical competencies. The health outcomes element consists of utilization of healthcare services, health status indicators, health severity indicators, adherence to recommended care regimens, and satisfaction.

The three elements are presented in a model (see Figure 1). Within the model, the background variables are viewed as interacting cumulatively, simultaneously, and often interdependently to produce a health behavior or behaviorally related health outcome; neither is viewed as an antecedent to the other. The dynamic variables, the motivation (the patient's behavioral goals and process for pursuing their goals), cognitive appraisal (knowledge, beliefs, 
and attitude toward an illness or treatment), and affective response (emotional response to a health concern), are influenced by the background variables, and are also viewed as mediators to one another and to health behaviors or behaviorally related health outcomes. Additionally, the model represents a continuous, reciprocal interaction between aspects of the patient's singularity (specifically dynamic variables) and the patient-provider interaction element. The relationships between and among the elements support the basic hypothesis of the model: as the provider interaction or intervention is tailored to the uniqueness of the patient, the potential for positive health outcomes increases (Cox, 2003). Thus, the content and process of the patient-provider interaction should vary according to the patient singularity expressed.

In the three decades since the theory was introduced, it has been used to by nurse scientists to guide numerous dissertations and other scholarly inquires. While Cox (1982) proposes that the IMCHB has many broad applications, one of which is to examine the efficacy of care approaches, researchers using the IMCHB have primarily focused on the relationship between the patient singularity variables and health outcomes. To date, only four studies (Brown, 1992; Cox \& Roghmann, 1984; Rice et al., 1994; Wagner, Bearn, \& Davidson, 2011) out of 29 manuscripts identified in the literature investigated the patient-provider interaction. Thus, the suggestion from an early critique of the model (Carter and Kulbok, 1995) to increase investigation of the patient-provider interaction element remains relevant.

Four studies were identified that included a military or veteran population (Carter, 1997; Garvin, 2012; Troumbley, 1988; Troumbley \& Lenz, 1992); none of the studies addressed the element of interest, the patient-provider interaction. There are no studies on women veterans guided by the IMCHB. However, the validity of the patient singularity element among women veterans is supported by the literature. Compared to civilian women, women veterans are unique 
with regard to their social and cultural influence, and the effect of such on their affective, motivational, and cognitive response to an illness (Strong, Crowe, \& Lawson, 2018). From basic training on, military personnel are indoctrinated with military ethos, or military values, which are reinforced regularly during one's military career (Center for Deployment Psychology, 2014). Military values include selflessness, loyalty, stoicism, and excellence. The values promote placing the welfare of others above one's self, a commitment to completing missions, an ability to endure hardships without complaint, and an emphasis on being the most effective and best professional service person possible (Westphal \& Convoy, 2015). However, these values may also indirectly make veterans vulnerable by contributing to the perceived stigma associated with help-seeking (Pease, Billera, \& Geard, 2015; Tanielian et al., 2008). When health issues present, service men and women may feel ashamed of their imperfections, may not acknowledge signs and symptoms of health problems, and may not place value on their own personal health.

Additionally, women veterans may also have deployment related experiences that are not experienced by civilians. These include combat exposure or MST which predispose women veterans to mental health issues (Hoglund \& Schwartz, 2014; Maguen et al., 2012). Stigmaassociated beliefs about mental health illness may impact women veterans' affective, motivational, and cognitive responses to a health concern (Resnick et al. 2012). Additionally, many VHA system (limited availability of women's health services) and environmental barriers (distant proximity to health care services) to obtaining needed healthcare exist for women veterans (Cordasco et al., 2016; Lahavot et al, 2013; Washington et al., 2011; Washington et al. 2015). As established earlier, health outcomes for women veterans are often worse than their male veteran and civilian women counterparts. Civilian provider lack of awareness of these 
patient characteristics and how to deal with them may negatively impact the patient-provider relationship and patient health outcomes.

Due to concerns regarding civilian provider preparedness to deliver care to veterans, interventions have been developed to educate nurses (Westphal \& Convoy, 2015), medical students, and residents (Ross, Ravindranath, Clay, \& Lypson, 2015) about military culture. Additionally, training courses are available to civilian providers to enhance their military cultural competence, knowledge of veteran health issues, and competency in evidence-based treatments (Center for Deployment Psychology, 2014; Koenig, Maguen, Monroy, Mayott, \& Seal, 2014). While the aforementioned efforts are important, the woman veteran's perspective is a decidedly missing component of determining the status of and interventions to improve civilian delivered health care to veterans. Thus, the purpose of the study was to understand the patient-provider interaction in civilian delivered healthcare encounters from the perspective of the woman veteran.

\section{Significance}

Several areas of significance are addressed by this study. First, many health disparities exist for women veterans. It is nursing's social responsibility to work to reduce these disparities and to provide quality care to this population. The disciplinary perspective of nursing centers around "...facilitating humanization, meaning, choice, quality of life, and healing in living and dying" (Willis, Grace, \& Roy, 2008, p. 32-33). Thus, the results gleaned from this study contribute to the body of nursing knowledge about caring for the woman veteran population to reduce disparities and aid in improving the quality of care delivered.

Second, concerns exist regarding the quality and effectiveness of civilian care delivered to women veterans, yet little is known about this issue from the perspective of the woman 
veteran. Thus, this study contributes to reducing the gaps in knowledge related to women veterans who seek care in the civilian sector. Additionally, results of this study inform the use of an extant nursing theory related to the content and processes of nursing care provided to and the subsequent health outcomes in a population of women veterans. Additionally, results will guide future research studies to further extend or validate the theory for this population. Finally, the results of this study may be used to inform patient-provider interactions during healthcare encounters with women veterans in the civilian sector, and ultimately, health outcomes for women veterans.

\section{Purpose and Definition of Terms}

The twofold purpose of this qualitative descriptive study was to describe women veterans' perspectives about interactions between themselves and civilian providers and to critically analyze the veterans' data to further inform Cox's IMCHB. The research questions were:

1. What are women veterans' descriptions of interactions between themselves and civilian healthcare providers?

2. How do women veterans' descriptions of the interactions between themselves and civilian providers further inform the use of Cox's IMCHB with the woman veteran population?

Definition of terms. Client-professional interaction (patient-provider)-the interaction between the patient and the provider defined by the following four components, which should vary according to the patient's singularity and health issue expressed (Cox, 1982)

Affective support-the provider's attendance to the patient's emotional response to a health issue and the development of an affiliative bond (Cox, 1984) 
Professional-technical competence-the ability of the provider to respond to the patient's technical and interpersonal needs, and to vary this response dependent on the patient's needs during a health problem (Cox, 1982; 2003)

Information giving- the quantity of, nature and content of information, and the manner in which information is provided to patients to impart knowledge about the threat of a particular health problem (Cox, 1982; 2003)

Decisional control-the provider's recognition of the patient's ability to participate in decision making about their own health care (Cox, 2003, p. 96)

\section{Method}

This investigator employed a qualitative descriptive methodology using directed content analysis to describe women veterans' perspectives about interactions between themselves and civilian providers and inform Cox's IMCHB. Qualitative content analysis is defined as “...a method for the subjective interpretation of the content of text data through the systematic classification process of coding and identifying themes or patterns" (Hsieh \& Shannon, 2005, p. 1278). One approach to content analysis, directed content analysis, is used when an existent theory about a phenomenon is incomplete or requires further elaboration (Hsieh \& Shannon, 2005). As noted above, more investigation on the patient-provider interaction component of the IMCHB is warranted. Further, more investigation is necessary to better understand the patientprovider interaction in the context of women veteran and non-VHA, civilian provider encounters. Qualitative descriptive methodology and directed content analysis are discussed in detail in Chapter 3. 


\section{Chapter 2: Literature Review}

Women veterans are returning from war with unique health needs which require ongoing care. Many obtain care from the civilian healthcare sector, outside of the Veterans Health Administration (VHA) care systems. There is a dearth in the literature about the healthcare received by women veterans from non-VHA civilian providers, and their subsequent health outcomes. The interaction model of client health behavior (IMCHB) is the theoretical lens used to view the interaction between a woman veteran patient and a provider. A review and synthesis of the literature, including the conceptual, methodological, and empirical evidence about the primary element of interest - the patient-provider interaction between veterans and healthcare providers - follows. The four factors that define the patient-provider interaction element of the IMCHB, decisional control, professional-technical competence, affective support, and information giving, guided the literature search.

\section{Search Strategy and Outcome}

A search of the literature was executed in the CINAHL, Medline, Academic Search Complete, and PsychInfo databases to obtain a better understanding of what is known about the interaction between women veterans and healthcare providers. Limits set for the search included English language, adult population, peer reviewed, and abstract available. The year of publication limiter was set from 2006 to 2020 to obtain articles most relevant to women veterans of Gulf War II or post-9/11 war eras. Starting in 2006, significant attention in the research literature was directed to the needs, barriers, and future research needed on women veterans who served in post-9/11 conflicts (Frayne et al., 2006; Vogt et al., 2006; Yano et al., 2006).

Using Booleen operators, several searches were employed using the advanced search tool to combine the primary key search terms (and synonyms) with secondary key search terms (and 
synonyms). The primary key search terms included women veterans, female veterans, service member, National Guard, and military. Secondary key search terms were derived from the IMCHB; specifically, interaction model of client health behavior, client professional interaction, decision making, education, communication, competence, patient provider relationship, encounter, therapeutic relationship, and provider. Additionally, the terms barriers, perspectives, and needs were combined with the primary key terms. Three separate searches yielded 538 manuscripts after duplicates were removed. All titles and abstracts were screen for relevancyinteraction (potential or historical encounter) between a veteran and a healthcare provider, VHA or civilian. While the focus of the review is the interaction between women veterans and healthcare providers, articles including men were not excluded. Full text manuscripts were obtained for the 25 manuscripts included in this review.

One additional search was conducted with the terms "interaction model of client health behavior" and military or veteran; the publication limiter was extended to 1982 (the year the model was first published). The search yielded four investigations in which the IMCHB was used to structure studies in a military or veteran population (Carter, 1997; Garvin, 2012; Troumbley, 1988; Troumbley \& Lenz, 1992). The primary aims in all studies were focused on the relationship between patient singularity variables and health outcomes, rather than the patient-provider interaction. Thus, all were excluded from this review.

\section{Literature Review}

It is important to note that all of the veteran participants in the aforementioned studies, except two, were recruited from VHA or military healthcare facilities. In one of the exceptions noted, participants included women veterans who had experience with one (or both) healthcare delivery systems_-VHA or community/civilian (Koblinsky et al., 2017). In the other, the data 
was collected by proxy informants versus veterans (Kotzias et al., 2018), The proxy informants were crisis line responders who had spoken to women veterans in crisis, who had used both VHA and non-VHA healthcare providers. In 16 of the studies reviewed, the participant sample included both male and female veterans, and in nine studies the sample included only women veterans; though one (as noted above) was collected by proxy informants for women veterans. Unless identified otherwise, the studies reviewed below include both male and female veterans.

Conceptual. A framework is the structural underpinning of a study and helps to identify the underlying rationale for the inquiry (Polit \& Beck, 2012a). When reviewing literature, it is important to identify the theoretical or conceptual orientation an investigator applies to his or her phenomenon of interest. A variety of frameworks were explicitly identified in six of the 23 studies in this review (see below). In several studies, a framework was implicit—alluding to a conceptual perspective, though not formally acknowledging or describing a framework in the background section (Bastian et al., 2014; Blonigen, Bui, Harris, Hepner, \& Kivlahan, 2014; Bohnert, Zivin, Welsh, \& Kilbourne, 2011; Eliacin, Salyers, Kukla, \& Matthias, 2015; Ganzini et al., 2013; Lederer et al., 2015; Kimerling et al., 2011; Kotzias et al., 2018; Phillips, McAndrew, Maharg, \& Bloeser, 2017). In eight of the studies no framework was identified (Abraham, Wright, White, Booth, \& Cucciare, 2017; Chase, McMahon, \& Winch, 2016; Ingelse \& Messecar, 2016; Koblinsky et al., 2017; Laird, Tolentino, \& Gray, 2013; Lee, Westrup, Ruzek, Keller, \& Weitlauf, 2007; Shamaskin-Garroway, Knobf, Adams, \& Haskell, 2018; Street et al., 2019).

A brief discussion follows for the five studies in which a framework was made explicit. Jeffreys, Leibowitz, Finley, \& Arar (2010) cited the transtheoretical model of health behavior change to situate their understanding of how patients move from trauma nondisclosure to 
disclosure in the healthcare encounter. Little discussion of the model was offered. In a study on disclosure of alcohol use in military veterans, Cucciare et al. (2015) identified use of the consolidated framework for implementation research. The authors reported using three domains of the framework: the outer setting (patient's social and economic factors), the inner setting (clinic structural, cultural context), and the characteristics of the providers involved in the patient-provider interaction, to structure the interview questions for their qualitative study. The Social Ecological Model was used by Balbale, Morris, \& LaVela (2014) to develop an interview guide to explore the individual-, environmental-, and system-level factors shaping veteran perceptions of patient-centered care.

Fischer et al. (2016) reported the initial qualitative component of a mixed-methods study. The authors noted that the veteran- and provider-specific interview guides were informed by the project conceptual model which combined two models--Pescosolido's network episode model and Fishbein's integrative model of behavior prediction and behavior change. No elaboration on either the two models, or the project model, was provided. Etingen, Miskevic, and LaVela (2016) used the patient centered care model to guide their study on the relationship between patient experience and quality of care. Trentalange et al. (2016) cited the Donabedian model as the structural guide for their study on the association between two provider characteristics, type of provider and designation as a women's health provider, and women veterans' satisfaction with care. The authors elaborated that the model describes the influence of patient characteristics, the structure of care, and the process of care on quality of care.

The theoretical model of patient-centered communication was used by Slatore et al. (2014) to guide a study to investigate patient and provider characteristics associated with highquality communication. The authors noted that important components in the theory include 
fostering the relationship, gathering and providing information to make informed decisions, enabling patients to manage their health, and responding to patient emotions. The aforementioned model was also used by Slatore, Golden, Ganzini, Wiener, and Au (2015) to guide the examination of the quality of provider communication on perceived risk and patient distress in patients with lung nodules.

Although a variety of theoretical frameworks aside from the IMCHB were used in the studies described above, many of the components of the theories parallel the major elements in the IMCHB. For instance, the Donabedian model described by Trentalange et al. (2016) includes the influence of patient characteristics, which is similar to the patient singularity element in the IMCHB. Additionally, the structure and process of care in the Donabedian model is related to the patient-provider interaction in the IMCHB. While quality of care is not necessarily an explicit element in the IMCHB, it is related to satisfaction with care (one of the variables studied by Trentalange et al., 2016) which is identified as a health outcome in the IMCHB.

The patient-provider interaction element of the IMCHB includes four factors, affective support, information giving, decisional control, and technical-professional competence. Each factor represents a role or action the provider demonstrates during the interaction with a patient. The patient-centered communication model used by Slatore et al. (2014) includes components that are similar to the factors in the patient-provider interaction; providing information to make informed decisions is representative of information giving, enabling patients to manage their health is representative of decisional control, and responding to patient emotions is representative of affective support.

Finally, the four factors of the patient-provider interaction element are intended to be tailored to a patient's singularity, including both the patient's historical-social-cultural-economic 
background and the patient's emotional-motivational-cognitive response to a health concern, to elicit or improve a behaviorally related health outcome. The premise is that without considering the patient's singularity, health outcomes may be less positive. In their study on women veterans disclosure of alcohol use (which can be considered a behaviorally related health outcome), Cucciare et al. (2015) used the consolidated framework for implementation research. One of the domains described, the outer setting (patient's social and economic factors), is related to the element patient singularity in the IMCHB model. Additionally, the provider characteristic domain described is related to the technical-professional competence factor of the patientprovider interaction element.

Methodological. This review includes studies from both qualitative and quantitative research traditions. Eleven of the studies included are quantitative; fourteen are qualitative. Among the qualitative literature the specific methodologies reported include: interpretive description (Shamaskin-Garroway et al., 2018), ethnography (Lederer et al., 2015), and grounded theory (Chase et al., 2016). In eight studies, the methodology was only referred to as qualitative (Abraham et al., 2017; Cucciare et al.; 2015; Eliacin et al., 2015; Ganzini et al.; 2013; Ingelse \& Messecar, 2016; Jeffreys et al., 2010; Koblinsky et al., 2017; Kotzias et al., 2018; Street et al., 2019). As noted previously, the authors in one study report presenting the qualitative component of a mixed-methods study (Fischer et al., 2016).

The majority of data collection in the qualitative studies reviewed occurred through inperson or phone interviews, using semi-structured interview guides. In two studies (Abraham et al., 2017; Koblinsky et al., 2017) focus groups were used. Finally, in one study (Balbale et al., 2014) the authors report employing Photovoice in addition to semi-structured interviews. In 
photovoice, participants are asked take photographs of meaningful elements which are then used by the researcher to stimulate dialog and prompt participant sharing of their narrative.

In the quantitative literature, only one study was described as a prospective cohort study (Slatore et al., 2015). The remaining studies were cross-sectional. In most studies, surveys were used as the method of data collection (Bohnert et al., 2011; Etingen et al., 2016; Laird et al., 2013; Lee et al.,2007; Phillips et al.,2017). In five of the studies reviewed, secondary data was analyzed. Blonigen et al. (2014) analyzed fiscal year (FY) 2007 and 2008 data from a national program evaluation of VHA mental health services. Data from the Survey of Healthcare Experiences for Patients (SHEP) was used in three studies; Kimerling et al. (2011) used FY 2007 data, and Trentalange et al. (2016) and Bastian et al. (2014) used data from FY 2013. Finally, in one study, baseline data from a randomized control trial was used (Slatore et al., 2015).

Empirical. All of the factors which comprise the patient-provider interaction element in Cox's model (affective response, information giving, decisional-control, and professional technical competence) were found in the literature review. Several factors were tied directly to a health outcome or behaviorally related health outcome as described by Cox $(1982,2003)$ (eg. satisfaction, utilization of healthcare services, or adherence).

Interestingly, in several of the studies reviewed, one or more of the factors that characterize the patient-provider interaction, though conceptualized or operationalized differently, were primary outcome variables. In addition, several of the factors were associated with a health outcome or behaviorally related health outcome as described by Cox (1982, 2003) (eg. satisfaction, utilization of healthcare services, or adherence). For instance, Blonigen et al. (2014) investigated perceptions of behavioral health care among veterans with substance use disorders (SUD). In one of their analyses, they examined which aspects of staff recovery 
orientation were independently linked to satisfaction with care. Items on their measure of staff recovery orientation are all related to decisional control; item examples include that staff: believed the patient could make their own life choices and listened to and respected the patient's choices.

As another example, Phillips et al. (2017) evaluated the effect of provider communication and interpersonal skills on veteran patients' satisfaction and treatment adherence. The survey used to measure communication included 12 items which are related to the information giving factor (eg. 'my primary care provider gave me clear instruction about my treatment for my MUS: what to do, when, how often, and for how long'). Also, there were four items on the survey about a provider's interpersonal skills which are related to the affective response factor (eg. 'my primary care provider is concerned about my feelings'). Other measures included satisfaction, past treatment adherence, intentions to adhere, and improvement in health in the preceding year. As a final example, in the secondary data analysis by Kimerling et al. (2011), the authors investigated veteran patients' perceptions of VHA health care quality. From the SHEP data, the authors selected nine dimensions of satisfaction for their analyses. Three of the dimensions are similar to factors in the IMCHB; education and information (information giving), emotional support (affective support), and patient preferences (decisional control).

The four factors of the patient-provider interaction element of the IMCHB are used to organize and present the synthesis of empirical results that follows. While several of the studies reviewed included one or more variables which are similar to the factors that characterize the patient-provider interaction, no studies included all of the factors. It is important to note that while each factor has been distinctively defined by Cox $(1984,2003)$, the factors are not necessarily distinct. Rather, there is an overlap or and inter-relationship between each factor, 
which is most likely due to the fact that they are each viewed as a component of an "interaction". In addition, as noted earlier, the variables in several of the studies are similar, but not a clear match to the factors as defined by Cox $(1984,2003)$. Therefore, results from the studies reviewed are grouped by the factor to which they were most closely related.

Affective response. Affective response is the provider's attendance to the patient's emotional response to a health issue or concern (Cox, 1984). The importance of a provider's affective response is evident in a study of women veterans who were asked to provide suggestions to improve both civilian and VHA mental health care (Koblinsky et al., 2017). The veterans suggested that providers should express empathy, promote dignity, and assure women veterans that they are worthy. Further, the importance of conveying a sense of worthiness, particularly to women veterans, was reiterated in a study to describe rural women veterans' use and perceptions of mental health services (Ingeles \& Messecar, 2015).

Street et al. (2019) studied women veterans' perceptions of military sexual trauma (MST) related communication. The theme of a provider's compassionate, non-judgmental stance was found to increase satisfaction with communication about MST. Having a compassionate, nonjudgmental stance included providers giving participants their undivided attention, listening, and believing them.

In four studies reviewed, factors that facilitate or influence disclosure of a health concern were investigated; disclosure of alcohol use (Cucciare et al., 2015), disclosure of suicidal thoughts (Ganzini et al., 2013), and disclosure of trauma (Jeffreys et al., 2010). There were similar findings with regard to the relationship of the provider's affective response, as perceived by the veteran, and veteran disclosure. Facilitators to disclosure were described as providers showing genuine concern, personal interest, and a caring attitude (Ganzini et al., 2013), being 
attentive, without being rushed (Cucciare et al., 2015; Ganzini et al., 2013), and demonstrating compassion and a hopeful attitude, and easing a sense of shame (Jeffreys et al., 2010). Barriers to disclosure included a provider perceived as judgmental (Jeffreys et al., 2010) or disrespectful (Ganzini et al., 2013; Jeffreys et al., 2010), uninterested, uncaring, rushed, or disingenuous (Cucciare et al., 2015).

The theme of respect and caring was evident in other studies as well. In a study about the inpatient hospital experience, women veterans reported overall satisfaction with care which was a result of being respected and cared for by attentive providers (Shamaskin-Garroway et al., 2018). Moreover, Fischer et al. (2016) found that respectful and caring providers enable some veterans to overcome their own attitudinal barriers (self-stigma, perceived weakness) to seek and sustain mental health care. In contrast, Chase et al. (2016) identified barriers to seeking care as providers who were perceived as dismissive or skeptical of a veteran's complaints, harsh or disrespectful, or questioned the accuracy of their symptoms. Finally, Koblinsky et al. (2017) noted that women veterans "... regarded the quality of the client-clinician relationship as a key driver in their decisions about whether to seek and continue treatment" (p.137).

Information giving. Cox $(1982 ; 2003)$ defined information giving as the quantity of, nature and content of, and manner in which information is provided to patients to impart knowledge about the threat of a particular health problem. The importance of the manner in which information giving occurs with veterans, as well as the nature and content of the information, is evident in the literature. Providers who provided thorough explanations were perceived as contributing to a patient-centered atmosphere (Balbale et al., 2014). Further, Shamaskin-Garroway et al. (2018) found that veterans voiced greater satisfaction when providers used understandable language and clear explanations. Slatore et al. (2015) evaluated the 
association of communication processes with distress and the perceived risk of lung cancer. Two instruments were used, the Impact of Event Scale (measurement of distress) and the Consultation Care Measure (communication measurement). Additional Likert scale questions were included regarding the patient's satisfaction with provider's explanation of the nodule, satisfaction with the care regarding the nodule, and perception of the provider's expertise in caring for patients with a lung nodule. The authors found that when veteran patients who have an incidental finding of a lung nodule and reported high quality communication with their provider, they experienced significantly less distress than those who felt ill informed or reported low quality communication with their provider $(\mathrm{OR}=0.28,95 \% \mathrm{CI}=0.08-1.00, \mathrm{P}=0.05)$.

As part of communication, the body language of a provider and the ability of the provider to listen are important in the exchange of information with veteran patient. In a study by Laird et al. (2013), the authors explored veteran patients' expectations during the greeting stage of medical visits. Results indicated that $61 \%$ of the veterans surveyed $(n=259)$ preferred a handshake at first greeting; only $17 \%$ did not, and $22 \%$ had no preference. Also included in the study was an open-ended question about veteran preferences at their first visit with a provider. The most frequent responses included smiling and being attentive. Chase et al. (2016) studied facilitators and barriers to care seeking among veterans at risk for traumatic brain injury secondary to blast-exposure. The authors reported that care seeking was facilitated when veterans experienced a provider who listened to them. Similarly, Cucciare et al. (2015) found that when providers took the time listen to a veteran patient's personal experience, veterans were more likely to disclose alcohol misuse.

Direct and indirect questioning, as well as less formal communication, in an atmosphere of empathy, facilitated disclosure of trauma for some veterans (Jeffreys et al., 2010). 
Additionally, providers demonstrating genuine concern and personal interest in a veteran facilitated disclosure of other, more difficult, health concerns (Ganzini et al., 2013). In contrast, in a study of veteran patients with chronic kidney disease (CKD), Lederer et al. (2015) found that when veterans felt rushed or disrespected, they limited sharing information with providers. Additionally, information sharing was limited when providers' discussions were too technical, too lengthy, or included scare tactics (Lederer et al., 2015).

Lederer et al. (2015) also reported that veterans desired that communication be tailored to their unique, individual needs. Similarly, in a study by Kimerling et al. (2011), the importance of tailoring communication to women veterans' unique experiences was highlighted. The authors investigated patient perceptions of health care quality among male and female veterans with and without a history of MST. Primary measures in the study included MST status, overall satisfaction, and nine other dimensions of care including: overall coordination, continuity, access, courtesy, education and information, emotional support, patient preferences, visit coordination, and specialist care. In post hoc analysis among the women veteran participants, after controlling for patient characteristics, satisfaction on four items related to education and information was statistically different for women with a history of MST, than for those without. The four items related to the provider delivering answers to questions, reasons for tests, results of tests, and side effects of prescribed medication in an understandable way. Women with a history of MST rated all aforementioned items lower than those without. The authors pointed out that greater attention to providing information, particularly for women veterans with a history of MST, is necessary. In another study about veterans' perceptions of satisfaction related to MST communication, Street et al. (2019) identified the theme of clear, concise interactions which promoted veteran satisfaction with communication about MST. Clear, concise interactions 
included the type of language used to screen for MST ("layman") and concise, brief interaction about the MST - participants appreciated that they were not asked to reveal extensive details of the MST during the interaction.

Phillips et al. (2017) evaluated the effects of a provider's interpersonal skills and communication with veterans regarding medically unexplained symptoms (MUS) on treatment behaviors and outcomes. High ratings of a provider's interpersonal skills, including being concerned about and understanding the veteran's feelings, and high ratings of a provider's communication increased veterans' satisfaction with care. Additionally, when providers held discussion with veterans about their MUS, increased treatment adherence and adherence intentions were associated with higher ratings of provider communication.

Similar results were found in a study by Etingen et al. (2016). The authors assessed the relationship between patient reported experience measures and aspects of quality of care. The patient experience included measures of patient perceived shared decision-making, provider caring (empathy, holistic care, and communication), respect for choices, and support. The authors found that veterans who saw providers that communicated well were able to more effectively manage their health. Additionally, veteran patients with effective health management also reported higher perceptions of shared decision-making, respect for choices, support, and higher participation in their own care.

Decisional-control. Decisional control is the provider's recognition of the patient's ability to participate in decision making about their own health care (Cox, 2003, p. 96). It is often the responsibility of the provider to communicate that patients have the right to participate in decision-making and to provide opportunity for such. Patient-centered care models have been implemented in many VHA facilities to increase patient participation and improve satisfaction 
and health outcomes. Balbale et al. (2014) explored patient perceptions and experiences related to patient-centered care initiatives. Patients reported that when care was perceived as being patient-centered, they felt informed and empowered, and felt a sense of responsibility to participate in their own care.

In the study by Lederer et al. (2015), veteran participants with CKD believed it was their responsibility to act as a 'listener' and trust providers to make their healthcare decisions. Eliacin et al. (2015) had similar findings in their investigation of veteran patients' preferences and appraisals of their involvement in treatment decisions. The authors reported that patientperceived provider expertise was cited as a reason for less involvement in decision making. Other reasons provided for less involvement included concerns about crossing a role boundary, fear of judgement, felling ill-equipped to manage their health, and concerns about health repercussions if they made a wrong decision. It has also been found that participation in decision-making may be further thwarted if providers use complicated explanations or medical terminology (Lederer et al., 2015), or appear to be focused on their own agenda (Jeffreys et al., 2010).

Blonigen et al. (2014) examined predictors of perceptions of behavioral health care among veterans with substance use disorder from a national program evaluation of VHA mental health services. Items selected as variables included satisfaction with care, helpfulness of providers, and perceived improvement. Specific measures of helpfulness included that providers: expressed high expectations for the patient's recovery, believed the patient could make their own life choices, listened to and respected the patient's decisions, asked about the patient's interests, helped the patient develop life goals beyond symptom management, helped the patient to include other important people in treatment planning, and introduced the patient to role models or 
mentors. The authors found that satisfaction was increased when patients perceived that providers listened, were recovery oriented (communicating hope and high expectations), and included them in treatment planning. In addition, other researchers have found that communicating support, respect for choices, and empathy, facilitated shared decision-making and may ultimately result in improved condition management (Etingen et al., 2016).

Professional-technical competence. The ability of the provider to respond to the patient's technical and interpersonal needs and vary this response dependent on the patient's needs during a health problem is the definition of professional-technical competence (Cox, 1982; 2003). Several of the studies reviewed indicated that provider competence is important to veterans during the patient provider interaction. In the qualitative study by Koblinsky et al. (2017), women veterans were asked to provide suggestions to both civilian and VHA mental health care providers. One of the prominent suggestions was that civilian providers screen patients for a history of military service. Without screening, the participants noted, an important part of a patient's clinical history is missing. The veterans also suggested that civilian providers obtain training in military culture and war related health issues.

Clinical training and proficiency with female-specific health care needs has been shown to increase satisfaction with care for women veterans. Within the VHA system, providers may be recognized as a designated women's health provider (DWHP) — primary care providers who are proficient and interested in women's health and are preferentially assigned to care for women veterans. Trentalage et al. (2016) examined the associations between two provider characteristics - class (nurse practitioner or physician), and designation, (DWHP or nonDWHP) — and overall satisfaction with the provider. Similarly, Bastian et al. (2014) examined the association between women veterans' experiences and provider designation, non-DWHP 
versus DWHP. The primary measure in the Bastian el al. (2014) study was overall satisfaction; secondary measures included access, communication, comprehensiveness, self-management support, shared decision making, and office staff. In both studies, when compared to nonDWHP's, satisfaction scores for DWHP's were significantly higher. Further, scores on comprehensiveness, communication, shared decision-making, and self-management support were significantly higher for DWHPs than for non-DWHPs (Bastian et al., 2014).

Also, in the study by Koblinsky et al. (2017), women veterans expressed a desire to have providers (both civilian and VHA) who are competent in alternative therapies and closely scrutinize medication use. Similarly, in a focus group study to explore the feasibility of a telephone delivered shared decision-making protocol for promoting alcohol behavior change, women veterans expressed the desire for providers to diversify treatment options to include alternative therapies (Abraham et al., 2017). Additionally, the scrutiny of medication use was reiterated in another study by Chase et al. (2016). Veterans with blast exposure (exposure to repetitive blasts during deployment to combat theaters) voiced the importance of a provider thoroughly investigating a patient's symptoms, instead of 'band-aiding' the symptoms with medication. Thus, provider vigilance in the pursuit of a diagnosis for a veteran patient's symptoms is important with regard to perceived competence, and impacts continued utilization of care. Finally, with regard to provider competence, Fischer et al. (2016) found that when veteran patients perceived that treatment was effective, trust in the provider increased and increased trust enabled veterans to overcome attitudinal barriers to mental health care.

In addition to technical competence, the importance of professional or interpersonal competence is evident in the literature on veterans. The ability of a provider to develop a quality relationship, communicate caring, and build trust with a veteran patient increased veteran 
willingness to disclose a health issue (Abraham et al., 2017; Cucciare et al, 2015; Ganzini et al., 2013; Jeffreys et al., 2010; Kotzias et al., 2018), increased care seeking (Chase et al., 2016;

Koblinsky et al., 2017), and facilitated sustained treatment (Fischer et al., 2016; Koblinsky et al., 2017). Recognizing diversity and demonstrating respect was fundamental to building a relationship with a veteran patient (Chase et al., 2016; Koblinsky et al., 2017). Additionally, for women veterans, communicating worthiness was essential to building a relationship with a woman veteran (Ingeles \& Messecar, 2016; Koblinsky et al., 2017; Kotzias et al., 2018).

Both the technical and professional (interpersonal) competence of a provider have important implications with regard to veterans' perceptions of a provider's ability to communicate. Slatore et al. (2014) reported using baseline data from a randomized controlled trial designed to improve communication quality with regard to end-of-life care in veteran patients with chronic obstructive pulmonary disease. The survey data utilized by the authors was completed by participants prior to the intervention. Participants were asked to rate their provider's general communication. The general communication domain of the Quality of Communication questionnaire was used and included six general communication attributes (using words you understand; looking you in the eye; answering all your questions; listening to what you have to say; caring about you as a person; and giving you his or her full attention); the communication score was computed as the mean score of the six attributes. Participants were also asked to rate their clinician's expertise in diagnosing and treating lung disease. The authors found that veteran-perceived provider expertise in treating lung disease was significantly associated with all communication attributes. Each one-point increase in perceived expertise was associated with an odds ratio of $2.10(95 \%$ CI $[1.52,2.88], \mathrm{p}<.001)$ with communication. 
With regard to interpersonal competence and communication, trust may facilitate communication. Bohnert et al. (2011) evaluated whether patient-rated measures of provider communication differed between patients with and without mental health diagnoses.

Additionally, the authors explored the moderating role of trust on the associations. Perception of provider communication was assessed via questions about the thoroughness of the provider's questions about the patient's symptoms and how they were feeling, the attention the provider gives to what the patient says, the provider explanations of the patient's problems or treatment, and the provider's knowledge about what worries the patient most about their health. Trust was measured via one question about how much the patient trusts their provider. The authors found that veteran patients with SMI or SUD were more likely to rate provider communication low when they experienced poor trust, but not when the trust was good.

Findings outside of the patient-provider interaction factors. Several other findings about the interaction between veterans and providers fell outside of the identified IMCHB theoretical factors but warrant discussion. The first finding is general and relates to the continuity of, accessibility of, and time with a provider. The second finding is specifically related to being a woman veteran.

Continuity, time, and accessibility. In several manuscripts, the continuity of the provider was indicated to be important. Increased satisfaction has been associated with an increased length of time seeing the same provider (Bastian et al., 2014; Street et al., 2019). During inpatient experiences for women veterans, having consistent nursing staff and regular doctors contributed to a more positive experience (Shamaskin-Garroway et al., 2018). Women veterans in the study by Koblinsky et al. (2017) voiced that having a continuous relationship with one health care provider was essential to building trust in seeking and sustaining treatment of mental 
health conditions. In addition, an increased level of engagement in mental health care has also been attributed to women veterans having a close relationship with a provider that was developed with repeated, consistent interaction with the same provider (Kotzias et al., 2018).

Cucciare et al. (2015) found that positive patient provider relationships encouraged disclosure of health issues, and that such were built on familiarity through contacts over time. In contrast, veterans in a study by Chase et al. (2016) identified that poor patient provider relationships were a barrier to seeking care in the VHA. Specifically, the veterans reported a high provider turnover rate at the VHA, subsequently every clinic visit in which they saw a new provider was like 'starting from scratch'. In the study by Ganzini et al. (2013), veterans expressed frustration when they saw multiple providers who repeated a suicide screening. The veterans felt that the repeated screenings conveyed the perception that the providers at the same health care facility do not communicate, and the providers did not take time to familiarize themselves with the patient medical record before the visit. Lack of a consistent provider and the repetitive screening contributed to discomfort and reduced trust, thereby producing a barrier to disclosure of suicidal ideation and seeking treatment. The importance of a provider, who is new to the patient, familiarizing themselves with the patient was also found in Laird et al (2013). Veterans voiced that they preferred that providers, at the very least, familiarize themselves with their chart prior to the interaction.

The importance of familiarity or continuity of a provider was reiterated by veterans the focus group study by Abraham et al. (2007). Veterans in the study noted that for a telephone intervention to be effective and true shared decision-making to occur, the caller should be a provider who has previously met the patient. Familiarity was essential to relationship building, 
especially in the context of discussing sensitive content in a seemingly impersonal manner (via telephone versus in person).

In addition to continuity of the provider, the perceived length of time spent with a provider and the accessibility of a provider appear to be important. An additional aim of the study by Trentalage et al. (2016), was to evaluate the mediating effect of patient perception of enough time spent with the provider on satisfaction with the provider. The authors reported that when veterans perceived that a provider spent enough time with them during a visit, a statistically significant increase in overall satisfaction with the provider was observed. Further, findings in multiple studies identified that when veterans perceived they were being rushed by a provider, disclosure of health issues and/or sustained treatment was not likely to occur (Cucciare et al., 2015; Jeffreys et al., 2010; Lederer et al., 2015). Finally, being able to check in with or access a regular provider increased a veteran's trust in the provider (Fischer et al., 2016; Jeffreys et al., 2010). The accessibility of a regular provider may be crucial to building trust with a veteran and important in overcoming barriers to sustained treatment in mental health.

Gender concordance. There is some evidence in the literature about women veterans that the gender of a provider influences the patient-provider interaction. With regard to communication with a provider, some women veterans reported not feeling comfortable talking about their health issues to a male provider (Cucciare et al., 2015). Similarly, for women veterans in a study by Shamaskin-Garroway et al. (2018), women providers were preferred as they were perceived as better communicators. One veteran participant in the study noted that she perceived that male providers try to pacify women or avoid asking questions, whereas women providers were more direct. It is important to note however, that in a study on women veterans, Bastian et al. (2014) found that satisfaction with care was not associated with gender 
concordance between a veteran patient and a provider. As discussed previously, when women veterans had a provider with specialized training in women's health (a DWHP) satisfaction was increased. This may indicate that while women providers may be more sensitized to women's needs in general, training may increase competency in that area for male providers.

For some women veterans, particularly those with a history of MST, the gender of the provider may be significant. Lee et al. (2007) explored the impact of clinician gender and examination type on women veterans' anticipated examination-related anxiety. All of the women veterans in the study had a history of MST. The study was used as a pilot to lay a foundation for future studies related to screening adherence and examination-related distress. Self-report instruments and a post-traumatic stress disorder checklist were used to collect the data. The authors reported that for women veterans with a history of MST, anticipated examination-related anxiety was significantly higher when the provider performing the exam (breast, pelvic, or rectal) was male $(\mathrm{p}<0.5)$. Similarly, in the study by Shamaskin-Garroway et al. (2018) one veteran participant noted that she had a preference for a female provider due to a history of MST involving men. The participant noted that despite the incident occurring 30 years prior, she still preferred a female provider.

\section{Summary}

This review of the literature supports the importance of the patient-provider interaction with regard to health outcomes or behaviorally related health outcomes for veterans. The quality of interactions between veterans and healthcare providers is vital to facilitating seeking treatment, sustaining treatment, treatment adherence, and satisfaction with care. Additionally, each factor identified by Cox $(1982,2003)$ as part of the patient-provider interaction was identified in the review. 
It is essential that the affective response of providers interacting with veterans can convey genuine concern, respect, empathy, caring, and attentive listening. Such is crucial to developing a relationship with the veteran patient. The interpersonal competence of a provider with regard to promoting dignity and worth, particularly for women veterans who report feeling less worthy than their male counterparts, and the building of trust, contributes to positive veteran-provider relationships. The necessary technical competence of providers caring for veterans includes, first and foremost, thorough history collection and screening, as well as knowledge of veteran culture and war related health issues. Provider vigilance in evaluating a health concern and pursuing a diagnosis, before treating symptoms with medication, is a main concern of veterans. Further, competence in traditional, as well as alternative, therapy options for veteran health issues, and close scrutiny when prescribing medications, is important to veterans. Finally, for women veterans, providers who are interested in and have special training in women's health issues are desirable and result in increased satisfaction with care.

There are varying desired levels of shared decision making among veterans. However, facilitation of shared decision making occurs when providers are patient-centered and communicate hope, high expectations, respect for veteran choices, and support. Additionally, veterans are more likely to participate when they feel included in treatment planning. Communication or information giving is a significant part of facilitating shared decision making and self-management of health. Information giving preferences of veterans include that the exchange of information be thorough, without complicated medical jargon, and communicated without the use of scare tactics. Additionally, veterans desire that there is mutual exchangewhere the provider also has the ability to listen, is unrushed, and attends to the veteran's personal experience, concerns, and questions. 
Two other findings in the review highlight areas that require further exploration. The first is the finding that seems to indicate that the continuity of, accessibility of, and time with a provider may have implications on the development of trust, and the facilitation of disclosure of health issues, continued utilization of care, and/or adherence to treatment. The second is that, for women veterans, the gender of the provider may have an influence on the patient-provider interaction, particularly when the woman veteran has a history of MST.

There are two significant gaps identified within this literature review. The first is that the majority of the literature reviewed included a mixed gender population. Only nine of the 25 studies were in a woman veteran population. Thus, women veterans in general are underrepresented in the literature. Secondly, all but one of the studies reviewed, both with mixed gender populations and with women only populations, have occurred with veterans who obtain care from VHA facilities. None of the reviewed studies explored the patient-provider interaction for women veterans who seek care from within the civilian sector. Considering that a significant portion of women veterans obtain care outside of the VHA health systems, from civilian health care providers who may not be familiar with military culture and/or screening for or treating war-related health issues, additional research is needed to close these gaps. This study contributes to reducing these gaps in the literature. Also, although some studies have addressed one or more of the factors included in the patient-provider interaction element of the IMCHB, none have addressed all of the characteristics. Thus, the applicability of the model to this population required further verification. Results of the study inform use of the model in future research with this population. For instance, the study results may be used to guide intervention development to support, strengthen, or improve the patient-provider interaction between woman veterans and non-VHA, civilian providers. 


\section{Chapter 3: Method}

There is a gap in the literature regarding women veterans' perspectives about health care received from civilian providers. A qualitative descriptive study was employed to investigate the research questions: 1 . what are women veterans' descriptions of interactions between themselves and civilian healthcare providers?; and, 2. how do women veterans' descriptions of the interactions between themselves and civilian providers further inform the use of Cox's interaction model of client health behaviors (IMCHB) with the woman veteran population? The following details the research design, including the sampling plan, method of data collection and analysis, and measures to protect human subject's rights. Further, measures to assure rigor are expressed in terms of credibility, dependability, confirmability, and transferability.

\section{Research Design}

It is the premise of this researcher that best practices for caring for women veterans come from gathering and understanding the individual's perspective. Thus, this investigator used a qualitative descriptive study design as described by Sandelowski $(2000 ; 2010)$ to address the research questions. Sandelowski (2000) states that "qualitative description is especially amenable to obtaining straight and largely unadorned.... answers to questions of special relevance to practitioners and policy makers" (p.337). Falling within a constructivist or naturalistic paradigm, the qualitative descriptive methodological approach takes into account that "reality is not a fixed entity but rather is a construction of the individuals participating in the research" (Polit \& Beck, 2012b, p. 12). A qualitative descriptive study design has the potential to uncover the complex and dynamic personal processes that occur in women veterans' health care experiences with nonVHA, civilian providers (Petty, Thompson, \& Stew, 2012). Given the lack of empirical evidence 
on women veterans' experience with non-VHA, civilian providers, a qualitative descriptive study is appropriate.

Cox's (1982) IMCHB (described in Chapter 1) is the theoretical framework that guided the study. The twofold purpose of this qualitative descriptive study was to describe women veterans' experiences of interactions between themselves and non-VHA, civilian providers and to critically analyze the veterans' descriptions to provide further information related to use of the IMCHB with the population of interest. Based on this purpose, the two research questions addressed by the study were:

1. What are women veterans' descriptions of interactions between themselves and civilian healthcare providers?

2. How do women veterans' descriptions of the interactions between themselves and civilian providers further inform the use of Cox's IMCHB with the woman veteran population?

\section{Protection of Human Rights and Ethical Considerations}

The Belmont Report articulates ethical principles that govern human subjects' research (Department of Health, Education, \& Welfare, 1979); these include respect for persons, beneficence, and justice. The principles are applied by attending to the following requirements: informed consent, risk/benefit assessment, and selection of research subjects. Prior to the start of this study, the research proposal, including informed consent process, recruitment plan, and demographic questionnaire, were submitted to the WVU Institutional Review Board (IRB). Considered a minimal risk study, the probability of harm or discomfort was deemed not greater than that encountered in daily life or during routine physical or psychological tests. Thus, the 
proposal qualified for an expedited review (WVU Office of Research Integrity \& Compliance, 2015); IRB approval was awarded October 19, 2018.

The informed consent process included the purpose and procedure for the study, contact information of the primary investigator, and a statement about the voluntariness of study participation (and ability to withdraw at any time). A waiver of informed consent was obtained for phone participants. However, the consent was reviewed word for word with each participant, whether the interview took place in-person or via phone. For in-person interviews, participants were asked to sign the consent. For phone interviews, consent was assumed if participants chose to remain on the phone.

Participant interviews took place in a private area and were recorded. At the end of the interview, participants were reminded that, as a voluntary participant, they could withdraw from the study at any point. Participants were offered contact information to mental health services in the event they experienced any psychological discomfort recollecting their health-related experiences. All participants declined information and denied psychological discomfort.

To protect a participant's identity, as interviews were transcribed, pseudonyms were used for health care facilities or providers that participants named. The recorded interviews were destroyed after the interview was transcribed. Data was de-identified by randomly assigning codes (\#0-12) on each participant's consent form, demographic form, and transcribed story. The consent and data forms were kept in separate locked cabinets in the researcher's office.

\section{Population and Sample Selection}

A purposive sampling plan was employed for the study. Purposive sampling ensures a relatively homogeneous sample, in which the participants have had adequate exposure to the phenomenon of interest (Cleary, Horsfall, \& Hayter, 2014). Participant selection must be in line 
with the specific purpose related to the research question (Collingridge \& Gantt, 2008) and utilize well-defined inclusion and exclusion criteria (Curtis, Gesler, Smith, \& Washburn, 2000). To be included in the study, participants had to be a female veteran, age 18 or older, who served during the post-9/11 or Gulf War 2 era, and who exclusively used non-VHA, civilian healthcare providers. Participants were excluded from the study if they were active duty, currently used VHA healthcare services exclusively, or were active dual users (use both civilian and VHA healthcare providers).

Participants for the study were initially recruited, beginning October 20, 2018, via West Virginia University (WVU) email, flyers posted at local grocery stores, primary care offices, and gynecology offices, social media postings (Twitter, Facebook), and postal-mailed recruitment letters. The recruitment emails were sent to WVU students who identified as a veteran; their email addresses were obtained from the WVU Registrars' Office. Postal-mailed recruitment letters were sent to women veterans who were accessing care at WVU Medicine, a local healthcare organization. Addresses for the women veterans were obtained through the WVCTSI database; a total of 100 letters were sent. A total of four participants responded to the recruitment email (two did not meet inclusion criteria), one to the recruitment letter, and six to the social media posting (three did not meet inclusion criteria). The remaining participants were recruited via word of mouth by participants referring their veteran peers.

With regard to sample size, too few participants may result in inadequacy of the breadth and depth of data gleaned, while too many may produce volumes of data that are unmanageable and superficial (Sandeloski, 1995). Sample size is considered adequate and sampling is ceased when redundancy is achieved—“"the conceptual wellspring has dried up and interviewees reiterate each other's ideas" (Cleary et al., 2014, p. 474). It is difficult to predetermine sample 
size in qualitative research (Flannery, 2016), but to achieve information redundancy within qualitative inquiry, 6-10 participants represent a generally acceptable starting point

(Sandelowski, 1995). In this study, redundancy was identified after 13 participant interviews, and recruitment was ceased on February 12, 2020. Redundancy was identified by a repetition of specific descriptors in data analysis.

\section{Data Collection and Analysis}

In a qualitative descriptive approach, gathering and analyzing data are conducted concurrently (Vaismoradi, Turunen, \& Bondas, 2013). Data collection and analysis for this study was guided by directed content analysis. When there is an existent theory or research about a phenomenon that is incomplete or requires further elaboration, a directed content analysis is appropriate (Elo \& Kyngas, 2007; Hsieh \& Shannon, 2005). In directed content analysis, the researcher begins by identifying key concepts from an existing theory or research as initial categories. Scripted interviews using open-ended questions, followed by targeted questions about the predetermined categories are used to collect data (Rubin \& Rubin, 2012; Wengraf, 2011). The unit of analysis for this study was the entire transcribed interview (Elo \& Kyngas, 2007; Graneheim \& Lundman, 2003).

Data collection. Veteran women interested in the study contacted the researcher by using the phone number or email listed in the recruitment letter, email, or the flyer/social media announcement. After being contacted by the interested participant, a full description of the study was provided, and a meeting was scheduled at an agreed upon location or via phone. At the meeting, the study purpose and procedures were reviewed. Once consent was obtained, participants were asked to fill out a demographic form (see Appendix A). 
After completion of the demographic form, the investigator began the interview using a scripted interview guide (see Appendix B). The strength of using a semi-structured/scripted questionnaire is that it helps to guide the interview and assure participants address the research questions identified (Wengraf, 2011). To avert a potential limitation, questions must be carefully worded to avoid leading questions which may bias the participant's response (Hsieh \& Shannon, 2005; Rubin \& Rubin, 2012; Wengraf, 2001). The interview script for this study consisted of open-ended questions about the predetermined categories: affective support, provision of health information, decisional control, and professional-technical competencies, which are elements of the patient-provider interaction described in the IMCHB. The questions were derived from the conceptual definitions of the predetermined categories. Open-ended questions were followed by targeted questions about the predetermined categories to probe more specifically about the provider's support, provision of information, decisional-control, and competency. In addition, interview-opening questions were developed to frame the narrative in relation to a particular health concern, and gain a better understanding of the participant's cognitive appraisal, motivation, and affective response at the time of the healthcare encounter. Questions regarding the outcome of the healthcare encounter and expectations for future healthcare encounters completed the interview.

Interviews were recorded and conducted in a private area to maintain confidentiality of the data. All interviews were conducted by the researcher to maintain consistency in data collection. Interviews lasted between 16 and 46 minutes (average interview length 34 minutes). Participants who completed the interview were given a \$30 Amazon, Walmart, or Sheetz gift card as compensation for time (and travel). The recorded interviews were transcribed word for word by the researcher immediately following the interview. 
Data analysis. Demographic data was summarized using descriptive statistics. After the interview was transcribed, the interviews were read several times to get a sense of the whole (Viasmoradi et al, 2013; Elo \& Kyngas, 2007). Coding of the data began with the predetermined categories of affective support, provision of health information, decisional control, and professional/technical competency. Because interview texts were several pages in length, and often dialog, though interesting, is not related to the research question, it was essential that the investigator kept the research focus in mind and searched only for codes that were relevant (Elo \& Kyngas, 2007). Any text that could not be categorized into the predetermined categories, yet were still relevant to the research question, were assigned a new code. After the transcripts were coded, specific descriptors from the text were extracted and placed into the categorization matrix under the corresponding category (Rubin \& Rubin, 2012; Schreier, 2013) (see Appendix C). Specific descriptors are manifest content lifted directly from each unit of analysis (Elo \& Kyngas, 2007). The specific descriptors were then divided into meaning units, which are “...the constellation of words or statements that relate to the same central meaning" (Graneheim \& Lundman, 2003, p.106). The meaning units were then shortened, using the process of abstraction, into condensed meaning units while still preserving the core meaning (Graneheim \& Lundman, 2003). Finally, the condensed meaning units were interpreted on a higher level to form themes (Vaismoradi et al., 2013). See Figure 2 for directed content analysis method for this study.

\section{Methods to Assure Rigor}

The concepts of credibility, transferability, dependability, and confirmability are applied to qualitative research to ensure trustworthiness (Elo et al., 2014; Lincoln \& Guba, 1985; Shenton, 2004). Credibility refers to the internal validity of the study or that the study design 
truly measures what is intended. Transferability refers to how the results of a study can be applied in other populations. Dependability refers to the ability to obtain similar results if the study were repeated in the same context, with the same methods and participants (Shenton, 2004). Finally, confirmability refers to the objectivity maintained in the study.

In this study, several strategies suggested by Shenton (2004), and others were employed to maintain rigor. First, credibility was maintained by using a well-recognized research method, directed content analysis. Directed content analysis is a well-cited method of analysis (Elo \& Kyngas, 2007; Hsieh \& Shannon, 2005), and is the “...strategy of choice in qualitative descriptive studies" (Sandelowski, 2000, p. 338). The second measure to ensure credibility is through debriefing sessions between this investigator and her Dissertation Committee. The Committee reviewed and approved the study prior to initiation, and monthly check-in points were held with the investigator's Dissertation Committee Chair across the project timeline. Finally, peer scrutiny occurred by a committee member who worked parallel to this investigator and randomly audited scripts, codes, and the categorization matrix for consistency of analysis.

To enable transferability, strict adherence to the established inclusion criteria was upheld, and a thorough description of the sample is provided in Chapter 4 (Graneheim \& Lundman, 2004). To ensure dependability and confirmability, a detailed description of all of the processes within the study - the sampling plan, method of data collection, and method of analysis is provided above. Additionally, an audit trail was maintained throughout the study. Documenting an audit trail, particularly through the analysis phase, provides assurance to other researchers “...that the work's findings are the result of the experiences and ideas of the informants, rather than the characteristics and preferences of the researcher" (Shenton, 2004, p. 72). Detailed notes documented on each unit of analysis (interview script) served as the audit trail. Finally, authentic 
citations (specific descriptors) from the data are provided via the categorization matrix to support the thematic interpretation (Elo \& Kyngas, 2007).

\section{Advantages and Limitations of Design}

There are advantages to the study's design. To this investigator's knowledge, this is the first study investigating the perceptions of a sample of women veterans who obtain care exclusively from civilian providers. Secondly, qualitative studies are well suited to provide rich contextual data about a phenomenon (Petty et al., 2012). Finally, this study was guided by a nursing theoretical framework. A theoretical framework identifies how a researcher is oriented to the phenomena of interest and increases the quality of the study (Polit \& Beck, 2012a). It is important to note that this study is not without limitations. The small number of participants and narrow inclusion criteria limit the application of the study findings to a larger women veteran population; however, the sample description is provided so that transferability to similar populations may be assessed. Finally, there is an inherent tendency for bias when using directed content analysis (Hsieh \& Shannon, 2005).

\section{Summary}

The qualitative descriptive study is one of many future steps to reduce the gap in the literature regarding civilian delivered health care to women veterans. To date, this is the first study on women veterans who exclusively use civilian delivered healthcare. A rigorous plan for sampling, data collection, analysis, and protection of human rights was detailed. Methods to assure the quality of and trustworthiness in the study were outlined. Results of the study provide women veterans experience of non-VHA, civilian delivered healthcare and inform use of the IMCHB to guide future research with women veterans. Finally, results of the study may be used to improve care delivered to women veterans by civilian providers. 


\section{Chapter 4: Results}

This chapter presents the results of the study Women Veterans' Descriptions of the Patient-provider Interaction with Civilian Providers. The theoretical framework, the Interaction Model of Client Health Behavior (IMCHB), used to guide the study was described in Chapter 2. In addition, the methodology employed in the study, qualitative descriptive methodology using a directed content analysis method, was described in Chapter 3.

A total of 13 women veterans meeting the inclusion criteria (not active duty, >18, using non-Veterans Health Administration (VHA), civilian healthcare providers exclusively) participated in the study. The mean age of participants was 38 (range 30-51), and mean years of service was 11.5 years. Greater than $80 \%$ of the sample was Caucasian. Ten identified as being married, two divorced, and one as single. All reported healthcare insurance; nine reported private insurance, two had Medicaid benefits, and two had Tricare. Participants resided in the following states, West Virginia, Virginia, Pennsylvania, and Florida.

\section{Background Variables}

The following branches were represented in the sample: Army, Army Reserves, Army National Guard, Air Force Guard, Air Force Reserves, Navy, and Navy Reserves. Some of the participants served in both active duty and reserves; one served in more than one branch. Four participants were commissioned officers, the remaining were enlisted. Eight of the participants were deployed one or more times, two were stationed outside of the United States, one reported a stateside assignment at a military medical center during the time of the conflicts, and two were not deployed. For those deployed, the range of length of time for a single deployment was 6-18 months. The participant roles in deployment varied; roles included nurse, physician, missile systems operator, medic, transport, specialist, security, and equipment recovery. Seven of the 
eight participants deployed identified that they were exposed to harmful agents during employment. Substances to which they were exposed to included: fuel, oil, smoke, insects, burn pits, dust, malaria, feces, urine, and asbestos. Of the eight deployed, seven completed a PostDeployment Health Reassessment (PHDRA) form. The PDHRA form is used to identify health concerns that emerge after deployment. Only one participant reported exposure to harmful agents on the form, one reported routine exposures, and five did not report exposures. None of the participants reported follow-up for exposure.

Three participants identified as having a service-connected disability. Eleven had previously sought care from the VHA. For those participants seeking care at the VHA, four reported that it took too long, or it was too complicated to obtain an appointment. As an example, one veteran stated,

"they say you can't make an appointment here, you have to go through here. And then, when you get there, you have to go there... So, it's like jumping through hoops just to try to get an appointment, and if you do get, an appointment, it's months down the road. And then, you're sitting there for hours".

Another veteran stated,

“...many people shy away from the VA because many people have had bad experiences there. I sincerely will not go to the VA. I tried one time to get an appointment, and they gave me the run-around. After that, I refuse to go".

Two female veterans described their experience attempting to obtain care at the VHA as "chaotic". Three participants reported that they were "turned away" because they "didn't qualify". One reported that she used VHA services for a brief time after separating from the 
military, until she was cleared from an illness scare related to deployment. One participant has access to VHA services but chooses to see civilian providers.

\section{Dynamic Variables}

Using the IMCHB as a guide, the researcher sought to add additional descriptions of the sample by identifying the dynamic variables of the participants. Dynamic variables are described by Cox $(1982 ; 2003)$ as the patient's cognitive appraisal (knowledge, beliefs, and attitude toward an illness or treatment), affective response (emotional response to a health concern), and motivation (the patient's behavioral goals and process for pursuing their goals). Most participants described cognitive appraisal of their health concern as "knowing something was wrong", with eight suspecting or identifying the concern as directly linked to their military service experience. Two reported that they needed a prescription or a prescription refill. Their affective response to the health concern varied from being "worried" to "concerned" to feeling "upside down". One participant shared that she felt she was "in a bad state of mind--desperate". The predominant motivation for seeking care was for worsening symptoms which were disrupting participants' everyday lives.

\section{Provider Characteristics}

The participants were asked questions about their healthcare provider's characteristics. The experiences described included both male (8) and female (5) providers, who were physicians (10) and advance practice nurses (3). Eight of the providers were generalists, and five were specialists. In addition, participants were asked about how each chose their provider. Five participants chose based on recommendations by peers or colleagues, two chose based on location, two by researching online reviews, three by seeking an in-network provider covered by their insurance, and one chose her provider because he was former military and a family friend. 
Finally, participants were asked about what their provider knew about their military service and how the provider came to know that information. Three reported that their provider was not aware of their military service, two reported that the provider knew very little, and nine reported that the provider knew "everything". Only one reported recalling a specific screening for military service (on an intake form), four could not recall if they were screened or self-disclosed, and the remaining reported self-disclosure of military service.

\section{Study Findings}

The predetermined categories of provider-patient interaction--affective support, decisional control, information giving, and professional/technical competence, were used as the organizing framework for presentation of the findings of the study. In answering the primary research question, what are women veterans' descriptions of interactions between themselves and civilian healthcare providers, several themes emerged as the findings. The themes include:

- Affective support is

o knowing me as a person by hearing my story and being attentive to my concerns.

- Professional-technical competence is

○ recognizing and acknowledging women are veterans, and

○ comprehending the military experience, and

o being thorough and accepting accountability for attending to a health concern.

- Information giving is

○ uncomplicated explanation that facilitates decision making.

- Decisional control is

○ collaborating by seeking input, providing options, and supporting decisions. 
See Table 1 for the themes and the associated condensed meaning units.

Affective support. When asked to describe how their provider responded to their concern about a health condition, all participants referred to the experience of being attentively listened to, in a non-rushed manner; of being heard. This is exemplified in the statement by one participant who said, “he wasn't on a computer or on a phone or anything. He looked me in the eye, he waited for me to finish speaking before he started. He didn't talk over me or interrupt me". Participants also perceived the provider as caring or empathetic when they were given the chance to explain their symptoms and discuss how it was affecting their life. A participant described an experience with her provider who, “...took his time, and listened to all my concerns. He didn't rush me at all, he actually really, truly listened to me... [he was] very understanding". Some participants shared that they had previous contrary experiences that were not positive; interactions they considered dismissive. For instance, one participant shared she experienced providers who, “...think they figure it out before you even had a chance to explain your symptoms”. And another stated, “...when I tried to explain to her what led to having the issue... she was like, 'well, it doesn't really matter how it happened'. And, it's like, well it does. ...I just want to feel like I'm being heard...".

Several participants referred to the availability or accessibility of their provider. One participant stated that her provider "...tries to accommodate the best he can.... if I need to be seen sooner for something". Others described their provider's timely response to phone calls, availability via paging for severe concerns, and commitment to help alleviate symptoms until an available appointment.

Several participants spoke of the desire to be recognized as a person, and not just as their health concern. One participant described it as a "big process" approach, and another as a "whole 
person assessment". Many endorsed wanting to share about what was occurring in their everyday lives, rather than just about the immediate problem. Several expressed that they appreciated it when an inquiry into their lives was made. One participant described it as,

“she asked how things are going. She doesn't just walk in staring at her computer and go, 'okay, what is specifically wrong with you?' She is just like, “okay, overall how are you doing? Is there anything else we need to take care of while you're here? ... [it made] me feel like I was a person".

Though most endorsed that they eventually self-disclosed their history of military service during the healthcare encounter; most weren't asked. This was perceived as a missing component of their provider's response to how they were responded to as a person. The participants regarded their military service as a significant part of their life - a part of their identity, a part of their story. One participant stated, "background... doesn't have to be the whole kit and caboodle... just some questioning and being able to let me tell my story... knowing about me". One participant shared that being asked about their military history helped them to "feel validated", and another described that it as "a dignity thing". In addition, being asked about their service history was important for many of the participants who appraised their health condition as being as a result of their military experience. One participant described that,

"if you go to receive health care for whatever reason it is, there's probably a story behind it... I'm entrusting you with my body and my care, and it doesn't make sense to me why somebody wouldn't want to know about the background how this problem arose. It's the need to feel heard".

Professional-technical competence. The majority of the participants made reference to not being asked about a history of military service. In addition, the majority of participants made 
reference to being discounted as a veteran because they were female. One participant stated, “you know, it could be because I'm a girl, or I'm a woman, I didn't get screened”. Further, a few described instances where they felt their military experience was dismissed as being not as hard as their male counterpart. This was summarized by the participant statement, "[he said], 'you didn't have to carry that much since you were a girl'. And, I was like, excuse me, I actually did!".

The participants acknowledged that their experience with civilian providers was often comparable to what they experience from the general civilian public. One noted she was used to the stereotype of, “oh, you're the veteran? Oh, I thought you were the veteran's wife”. Another participant statement captures the theme well,

“...they don't realize that a female can do or are put in the same types of environments as men when they're put overseas. ... I did go to war; I went through this stuff. It's not right just because I'm a girl or woman, doesn't mean that I couldn't have served, couldn't have went overseas, I couldn't have had these issues".

Most participants described that they felt their provider had a limited understanding of the military because of lack of exposure in the area that they lived. This was summarized by one participant when she stated, “...the civilian doctors here probably don't have any one-on-one with veterans or anybody that served in the military or aren't aware of incidences or accidents that we would have had if we're on full-time". However, four of the participants described experiences with providers who were former military (area close to a large military base), who were more understanding of their military experience. One participant captured this as, “...they get it if you were to tell them about where you were stationed. They'd be able to say, 'that happened to you and this is why'. ...they are familiar with things about the military and deployments and stuff'. 
Several participants also described that their providers did not connect their health concern to their military service. As one participant stated, "nobody has ever made the link. Like, I now have [medical problems] that I didn't have beforehand, but I've had ever since. Nobody has ever asked or made a connection". Participants also explained that not being able to make the connection between military service and a health concern, might affect treatment planning. As one so aptly described,

“...[being] sensitive or aware due something that might have happened in their background, is important. In case other things come up, you can then ask more things or go into more detail or provide resources. You don’t have to know every detail, but enough that you have awareness for future decision-making".

As noted earlier, the majority of participants made reference to not being asked about a history of military service. Most participants expressed that by not asking, the provider's assessment was hindered, and vital information related to their care was missing. This was best captured by two participant's statements. One stated,

“...knowing the experiences and screening people are important because if you look at me at face value, you wouldn't really necessarily assume I was at the height of doing wartime stuff... taking care of 19 year olds who were blown up and missing half their parts when they woke up".

Another stated,

"...there are things that happen to patients, that may be the last thing that you're thinking of, because maybe they come in for, I don't know, like insomnia, and that could be related to some really deep-rooted stuff—and you may not realize it if you don't know their history". 
Several participants made reference to a provider familiarity with their health concern, repetitive interaction with a provider, and a long-term relationship with the provider that added to their perception of the provider's competence. One participant stated, “...be aware of my problems, what my meds are..." so that she didn't "...have to go in and explain everything and start from scratch every time". Another described the importance of continuity with a provider, stating, “...it's really important because you can't always be the judge of how you were [the] last visit, compared to this one. Sometimes you think you're doing great, but from another's perspective they might pick up on something that you didn't”.

All participants made reference to their providers' thoroughness in investigating their health concern with testing. One stating, “...he did an entire panel of stuff, everything, just to have a basis for what was going on”. Further, many described that their provider made every effort to treat them but would refer appropriately if it was beyond the provider's expertise. This was summarized by one participant's statement: “...he knew he was going to have to send me to someone else, but he wanted to see what he could do in the meantime for [my severe health symptoms] at the time".

While participants described the competency of their providers to investigate and address their health concerns, they also described a limited provider-knowledge about services available from the VHA and a lack of understanding of eligibility for VHA services. A participant illustrated the lack of understanding when she described overhearing a provider talking about a veteran patient. She stated,

“somebody said, 'why don't they just go to the VA?' ...they may not have a serviceconnected disability... or, have you checked into it? There's a reason that they're here, not 
everybody has benefits, and not everybody has been screened. It is a very frustrating complex machine and it's not designed to meet everybody's needs".

Another participant described that it was no surprise that providers have limited knowledge of VHA services, stating, “...knowledge of what is available at the VA is terrible. Because I don't know how; I didn't know that I could get a VA card that says that I'm a veteran, for example. ...I had no idea".

Information giving. When asked to describe the information provided to them during the healthcare encounter, participants made reference to the importance of knowing about what was going on with the health concern. This included knowing about the condition, what was causing it, the rationale for testing, what to expect, and options for treatment. Several participants described that knowing this information helped them to feel calm and make decisions. One participant described it as: “...explaining what we are going to do, but also what potentially caused it... It just reassured me and helped me make the decision about what avenue to go to treat it." It was reiterated by another participant who stated that her provider will, “...pull up your testing in front of you and go over the results, showing the different aspects of the test and what is wrong. Going over the testing results in detail and then talking about the possibilities of treatment, giving me all my options. It helps me to be a part of the decision-making”.

Participants also described that information was provided in an understandable way. One stated: "he used very simple terms that I understood and [I] could understand the process of how he got to the diagnosis". Participants described efforts to translate information that was difficult to understand. One description was, “....if I didn't understand it when she initially explained it, she tried to rephrase it so that I can understand. Or, she gave an analogy". Many also described 
the importance of how the information was delivered, one citing the "he did not rush". Others stated that they did not prefer "a lecture", to be "bullied with points", or a "barrage of information".

In addition to information provided during the healthcare encounter, all participants made reference to the importance of more information, information that could be referenced at a later date to aid in self-management of their health. One stated, “...he actually gave me a little pamphlet with several websites on it so that I could continue researching on my own time". Not all participants described that additional resources were available, but wished it was so. This is exemplified by one participant's statement, “...sometimes you get busy and you don't really search for it. But if it was handed to me, at the moment when we discussed everything, I think I would be able to more likely follow suit". Several participants identified the preference for a written pamphlet or a website.

Decisional control. When asked to describe the level of participation that they experienced during interaction with their provider, all participants made reference to the fact that they wanted to have a say or be involved in planning their care, and that they assumed responsibility for and knowledge of their own body. As a participant stated:

"I want the most control because it's my body. I don't think that just because they're a doctor means that they know always what's best for me. So, I want all of the information so that I can make the decision. Doctors are people too and I think some people see doctors as.... deities, but they are just people." One participant described being supported by her provider to be involved as, "[giving me] the different options, and allowing me to make a decision, and then be fine with my decision". 
Another participant shared how she felt about a previous experience in which she did not feel she was permitted to participate, stating, “...it's like they didn't respect me to make a decision. So, it's like a level of arrogance. It's a lack of respect. ...insinuating that I can't think for myself'.

Also, participants consistently described the desire to have a partnership with their provider. Many referenced being part of a team, working with their provider, or working together. Several contrasted their experience of being in a partnership with their non-VHA, civilian provider to their military experience. One participant noted the difference as: "...cause in the Army you are told, 'hey, this is what you are taking, this is what we are gonna do"'. Another described, “...building that team and the partnership and not having that chain of command where 'I'm the doc, and you're not'".

In addition to working together, participants described the importance of their provider knowing their goals. One participant stated that her provider "understands that what I want to do is really important to me". Another exemplar is observed in the participant statement, “...work with me and figure out how I can do the things that I'm trying to do. If you're working with me to help me get to do what I want to do, that means you understand how important that thing is to me".

Health outcomes. The hypothesis underlying the IMCHB, which was used to guide the study, is that as the provider interaction or intervention is tailored to the uniqueness of the patient, the potential for positive health outcomes increases (Cox, 2003). Thus, it was important to capture the participants' descriptions of the overall outcome of their interaction with a nonVHA, civilian provider and the outcome of their encounter. Overall, the participants interviewed described outcomes of their experiences with their current non-VHA, civilian health care providers positively. All of the participants identified that their need was addressed sufficiently 
at or above their expectation. When questioned about how they wished future visits with their provider would go, many said that they would prefer it to continue as is. All participants reported that they planned to continue to see their current provider; in fact, one participant stated, "I wouldn't trade him for the world".

One participant noted that while her needs were met, she wished her provider would have screened her for military history and asked more questions about why she was on medication. She went on to note that while her health condition was stable, if it were not, or another veteran who had a traumatic military experience saw that provider, the outcome might have been different, or "severe". There were some instances during the interviews where participants briefly referred to a negative experience with a provider. For example, when a provider discounted their service experience as a woman, "blew off" their concern, or was not fully addressing their healthcare need adequately, many reported switching to their current provider. However, one noted that after her concerns were dismissed, she never went back to that provider or sought additional help for that health care concern. She noted that she chose to manage the health concern on her own, and that for a period of time the concern was "mostly stable". It wasn't until later, during an encounter with a different provider, for a separate, unrelated concern, that her original health concern was addressed. The poor affective support of the first provider resulted in delayed treatment for a health condition. Another described being "lectured to" by a provider about an abnormal lab result which resulted to her switching to her current provider. Thus, the manner of providing information contributed to dissatisfaction.

Additional findings. At the conclusion of the interviews, participants were asked about which of the provider's behaviors were most important to them, the provider's response to their concern, the provider's skill, information, or involvement in the treatment plan. Participants 
indicated that decisional control was the most important. The second most important was the provider's skill, and the third, the provider's response to their concern.

When asked about suggestions for non-VHA, civilian providers, almost all participants responded. Overwhelmingly, they endorsed the importance of screening for military service and experiences, and having adequate knowledge of the military experience, including exposures and subsequent health risks related to deployment.

Some participants spoke to the reality of veterans obtaining care in the civilian sector. One stated, "I think if they're going to be a provider, it doesn't really matter where ever they're located at, there's a point in their career they're going to come across a service member that's going to have, you know, service related issues." And another drew attention to recent legislation intended to increase healthcare access to veterans, “... you know the MISSION act just passed. So, there's going to be a lot more veterans who are going to be seen in community settings. So, it's probably time for them to have more information".

As for the 'more information', the participants indicated they wanted providers to know “...more about the military, ...more about some of the most common ways that military service can affect the body and mind". In addition, to gain knowledge about exposures that occur during deployment. One participant shared,

“...there's a lot of women out there who have been deployed who did get hurt or traumatized or have PTSD. I mean, they may not have been in the infantry, but they still had exposures. Like for example, if they were a truck driver. You don't have to be in the infantry and still see combat".

Also, some participants noted the importance of knowledge about where or when a veteran deployed. For instance, one participant made the point that "if you got out in 1998, it 
would be much different than if you are a female getting out today. Most of them [referring to the latter] deployed to a combat area". Another highlighted that different exposures occur in different areas,

“...if you were deployed to Kuwait, Iraq, or Afghanistan, [you might have been exposed to] ...the burn pits... ... as far as chemicals, there's no specific chemical that they might be exposed to, more so like a sandstorm, you're breathing in all the sand".

Participant rationale for screening was varied. Many of the participants indicated that the importance of screening is because many veterans may have experienced traumatic events during deployment, “...sometimes they're exposed to military sexual trauma or... ...exposed to things like seeing other soldiers getting killed or IED explosions", that may negatively affect mental health. Several participants noted that many veterans do not know to share the information or are reluctant to bring it up because of stigma. One stated, “[we] aren't going to speak up because we don't want to be seen as the weak link". For those who are still serving (referring to Reservists or Guard), one veteran reported that it might not be shared due to fear it will affect his or her career, stating,

"I have friends who are still more active and they will not seek help because they're afraid it'll get back to the military and they won't be able to fly. If you have insomnia or nightmares — things happen over there — and you just don't talk about them. ...[if] it gets back to the military and they think we can't do our job. ...it could potentially destroy a career. I believe that you should screen because veterans hide things. And, you need to know, that as a provider, any veteran who has any deployments, any deployments, is hiding something”. 
A few participants offered example screening questions. One suggested, “...an open question like, 'is there anything you would like to share with me that may have affected your condition, due to service?' Make it [the question] empower the patient to be able to say things". Another suggested,

“...ask what branch, and what did you do in, or what does that job entail? Explain that to me, is it similar to this or this in the civilian world? Or ask: do you want to share if there was any experience in your service or when you deployed? Or about their support network".

However, one participant indicated that if a provider was merely adding a screening to complete a checklist, without knowledge of the military, military health risks, or appropriate treatment plans or resources, it is not necessary. She posed, “...what's the end goal? Is it just another piece of data? Is it going to be something that alters what you do?" This was expanded on by another veteran who stated, “...if they don't understand these issues that are coming to them in a different form, you have a broken treatment plan".

\section{Relation of the Themes to the IMCHB}

The second question in the study was related to how women veterans' descriptions inform the use of the IMCHB. As the data were analyzed, no additional categories related to the patient-provider interaction were identified. All of the specific descriptors lifted from the text fit well into the predetermined IMCHB categories. The rich descriptions from the participants provided meaningful units from which the themes for each of the categories emerged. The themes provided a unique female veteran perspective about each factor of the patient-provider interaction element of the IMCHB. 


\section{Summary}

The research questions posed for this study were "what are women veterans' descriptions of interactions between themselves and civilian healthcare providers", and "how do women veterans' descriptions of the interactions between themselves and civilian providers further inform the use of Cox's IMCHB with the woman veteran population?’. Employing a qualitative descriptive methodology using directed content analysis for which the IMCHB operated as a guide, five themes emerged to describe women veterans' experience with non-VHA, civilian providers. In addition, the themes inform the future use of the IMCHB by illuminating the unique perspective of women veterans about each factor in the patient-provider interaction element of the model. The resulting themes for each factor have implications for research, practice, and policy related to the care of women veterans. 


\section{Chapter 5: Conclusions}

This chapter presents discussion surrounding the findings of the study Women Veterans' Descriptions of the Patient-provider Interaction with Civilian Providers. To review, the study was guided by the Interaction Model of Client Health Behavior (IMCHB) (Cox, 1982; 2003). There are three main elements in the theory, patient singularity (which include dynamic and background variables), the patient-provider interaction (interaction between a patient and a healthcare provider), and health or behaviorally related health outcomes (utilization of healthcare services, health status or severity indicators, adherence to care regimens, and satisfaction). Four factors make up the patient-provider interaction element: affective support (a provider's attendance to the patient's emotional response to a health issue and the development of an affiliative bond; professional-technical competence (the ability of the provider to respond to the patient's technical and interpersonal needs); information giving (the quantity of, nature and content of information, and the manner in which information is provided to patients); and, decisional control (the provider's recognition of the patient's ability to participate in decision making). The hypothesis of the model is that as patient-provider interaction factors are tailored appropriately to the patient's unique singularity variables, increased in positive health outcomes are more likely.

\section{Primary Aim}

The first question in the study was: What are women veterans' descriptions of interactions between themselves and civilian healthcare providers? Women veteran participants were asked to describe their interaction with a non-VHA, civilian provider. Analysis of their descriptions resulted in rich themes about the four factors in the patient-provider interaction element. For this sample, affective support of a provider is about knowing me as a person by 
hearing my story and being attentive to my concerns. The professional-technical competence of a provider is about recognizing and acknowledging women are veterans, comprehending the military experience, and being thorough and accepting accountability for attending to a health concern. Information giving is about uncomplicated explanation that facilitates decision making, and decisional control is about providers collaborating by seeking input, providing options, and supporting decisions. The relationship of these themes to the existing literature are presented later.

While some of the themes appear to be similar to what any patient (civilian or veteran) might expect in a patient-provider interaction, like that of information giving (uncomplicated explanation that facilitates decision making), some are very unique to a woman veteran. For instance, to provide affective support to a woman veteran, it is important for the provider to know them as a person and be attentive to their story, not just their presenting health concern. A woman veteran's military service is part of who they are as a person and may have affected their health. Being able to tell their story to a provider who will attentively listen is important to getting to know them as a person and how their health concern is affecting them.

The themes related to professional-technical competence are also unique to a woman veteran. The theme of recognizing and acknowledging that women are veterans is important. Recognition is about screening patients — not just male patients - for a history of military service. Acknowledging their service history is about not discounting their service as less than that of their male counterparts simply because they are women. Comprehending the military experience is about understanding what experiences women veterans might have had while serving and what role they might have played while they were enlisted or deployed. 
The theme of being thorough and accepting accountability for accepting a health concern, at first glance appears similar to what any patient might expect (civilian or veteran). However, the theme is not only about attending to a health concern by investigating a problem and referring to a more specialized level of care if needed. Being thorough is also about considering military history during evaluation of a health concern. For women veterans, exposures during service may be linked to a presenting health concern and may be missed if the experiences are not accounted for during evaluation.

The theme of collaborating by seeking input, providing options, and supporting decisions also appears to be similar to what may be important to any patient. However, for a woman veteran it is unique because it is about partnering with a healthcare provider to come up with a treatment plan that incorporates their goals. It is about having options from which they may choose, and when they have chosen, their choice is supported. This expectation was contrary to what they experienced while in the military. For instance, one participant shared that she was "made to get on birth control" when she was deployed; she did not have a choice to decline.

\section{Secondary Aim}

The second question was: How do women veterans' descriptions of the interactions between themselves and civilian providers further inform the use of Cox's IMCHB with the woman veteran population? An early critique of this model indicated that few empirical investigations had occurred surrounding the patient-provider interaction element; the majority of empirical support involved the relationship between patient singularity and health outcomes (Carter \& Kulbok, 1995). Further, during the literature review, only four studies that were guided by the IMCHB occurred in a population of military or veteran participants, and those, like many other studies guided by the model, focused on the relationship between patient singularity and 
health outcomes (Carter, 1997; Garvin, 2012; Troumbley, 1988; Troumbley \& Lenz, 1992).

Thus, this study, though small, adds a much needed and unique extension to the model. First, there was coherence between the results and the model. No additional factors outside of those defined in the IMCHB were identified in the participant descriptions of their interaction with a non-VHA, civilian provider. Second, the findings seem to align with the hypothesis of the theory that tailoring the factors to the unique singularity variables of a woman veteran patients are important for improved health outcomes.

The focus of this study did not surround participant outcomes. However, questions about outcomes were included to provide contextual information to more accurately interpret the results related to the utility of the model. Participants' descriptions of their outcomes seem to indicate that when the patient-provider interaction factors are tailored to the uniqueness of a patient, the outcomes may be more positive. In addition, when the factors are not tailored, the outcomes may be less favorable. For instance, most participants described their provider's affective support positively, resulting in increased satisfaction (an outcome identified in the model) with their provider. In contrast, one participant described a provider's information giving as being "lectured to" which resulted in her switching providers (satisfaction). Another participant described an interaction in which the affective support of a provider was considered dismissive, and the resulting outcome was delayed treatment (poor healthcare utilization). Further, as many participants noted, if a provider's professional-technical competence is lacking with regard to being thorough (not accounting for military experiences), especially for women veterans with more traumatic military exposures, a "broken treatment plan" may result and in turn, more severe health outcomes (increased severity of health issue). 


\section{Relationship Between the Findings and Existing Literature}

Many barriers to VHA care for women veterans have been identified in the literature, including lack of knowledge about eligibility status and services (Washington et al., 2015), affordability (Lahavot et al;, 2013), and inability to take off time from work and care giver responsibilities (Cordasco et al., 2016). The results of this study add to the known barriers to obtaining VHA care for women veterans. These include the lengthy amount of time to get an appointment with a provider, the complicated experience of seeking care (described as "jumping through hoops"), and being evaluated as ineligible. Ineligibility prohibits women veterans from obtaining VHA services. However, despite three participants being eligible for VHA services, through a service-connected disability, they chose to see civilian providers. Reasons for their choice were not fully explored during the interviews.

Provider type. The providers who were described by participants in the study included both physicians and advance practice nurses. A preference for provider type was not identified by the participants. The only provider type preference found in the literature review was that of VHA providers who were recognized as designated women's health providers (DWHP)— providers who are proficient and interested in women veterans' health (Trentalage et al., 2016). Satisfaction with care was not associated with provider type (APRN or physician), but was significantly higher for DWHPs than with non-DWHPs. This designation may indicate a higher perceived professional-technical competence of a provider. The requirements to obtain this designation may be of relevance for non-VHA, civilian providers with regard to continuing education to become more proficient (increase professional-technical competence) in the care of women veterans. 
Gender concordance. Gender concordance was one of the findings that fell outside of the patient-provider interaction factors during the literature review. Women veterans with a history of military sexual trauma (MST) may prefer a female provider, particularly if the MST was perpetrated by a male (Lee et al., 2007; Shamakin-Garroway et al., 2018). The providers who were described by participants in the study included both male and female providers. A preference for provider gender was not consistently observed in the data. However, one of the 13 participants stated that, if possible, she avoided male providers, but did not elaborate beyond that statement. Additional research exploration for women veterans who have experienced MST, and who obtain care from within the civilian sector may be necessary.

Screening. Out of 13 participants, only one was asked by the provider about her service history. This is consistent with the literature about the readiness of non-VHA, civilian providers to care for veterans. A lack of consistent inquiry into military service history has been reported by others (Kilpatrick et al., 2011; Miller, Finn, \& Newman, 2014; Mohler \& Sankey-Deemer, 2017; Maiocco et al., in press, Maiocco et al., 2018). In one study that included nurse practitioner, physician, and physician assistant participants $(n=102)$, fewer than $57 \%$ screened for military service (Vest et al., 2018a). The lack of screening raised concern for participants in this study. Their concern echoes that of other women veterans (Koblinsky et al., 2017).

Military culture. From basic training on, military personnel are indoctrinated with military ethos, or military values, which are reinforced regularly during one's military career (Center for Deployment Psychology, 2014; Meyer, 2015). These values include selflessness, loyalty, stoicism, moral code, social order, and excellence. The values promote placing the welfare of others above one's self, a commitment to completing missions, an ability to endure hardships without complaint, following an internal moral compass and choosing right over 
wrong, finding purpose in defending society, never leaving a fallen comrade behind, and being the most effective and best professional service person possible. These values influence how veterans interpret and act on health care concerns (Convoy \& Westphal, 2013). For instance, expressing concerns about physiological or psychological symptoms is contradictory to enduring hardships without complaint or excellence, and may be self-assessed as being weak (Ingelse \& Messecar, 2016; Koblinsky et al., 2017). This sentiment was echoed in the participants stories. While most of the participants identified that they were comfortable seeking care, many spoke of what they perceived as a reluctance of their veteran peers to talk about health issues. They related this reluctance to the fear of being perceived as the "weak link", or the fear that by sharing, his or her military career might be impacted.

It must be noted that the influence of military ethos was also reflected in the participants' suggestions for non-VHA, civilian providers; specifically, the value of "leaving no man behind", which speaks a veteran's concern for his or her peers. The suggestions by participants to improve provider practice, including screening all patients and having more knowledge about military related health issues and symptoms, were not based on self-concern. Rather, the rationale for their suggestions was to prevent poor outcomes for their 'brothers and sisters' who may have had worse or more traumatic experiences compared to them. Such is a testament to their continued service to others.

All participants seemed eager to share their experiences for this study. However, it must be mentioned that despite multiple recruitment strategies, more than half of the participants were recruited via word of mouth by a veteran peer who had participated in the study. This may be a reflection of the influence of military culture for the participants_-relying on and trusting their 
fellow soldiers. An extension of trust that a research study has value or merit, or is worthy of participation, may be given more to a military peer than to a non-veteran researcher.

Patient-provider interaction factors. In the literature review, the factors, while clearly defined in the model, did not always appear to be distinct. There was an overlap or interrelationship between the factors, which is most likely due to the fact that each is a component of an "interaction". While the themes of the study emerged distinctly for each factor, an interrelationship between the factors was observed in the participant descriptions. For instance, information giving was about uncomplicated explanation that supports decision making. When participants were presented information in an understandable way, they were able to more thoroughly make decisions about their care. Without such, it is possible that participation in decision making would be thwarted (Lederer et al., 2015). Further, when information was provided in an understandable way, one participant described feeling reassured. Similarly, in a study by Slatore et al. (2015), when veteran participants perceived they were well informed about their pulmonary nodule, distress was reduced.

A relationship between decision-making and affective support was also observed. Participants described the importance of the provider seeking input about their personal goals. This might come up as a provider is getting to know them as a person while listening to their story. In other words, when a provider gets to know the woman veteran patient by hearing their story, personal goals might be identified and therefore integrated into planning potential treatment options. In addition, by hearing the participants story, providers would be able to include military experience in their diagnostic reasoning (relationship to professional-technical competence themes). 
Affective support. In the literature review, the dimension of time and accessibility did not appear to equate with any of the patient-provider interaction factors. In this study, when participants were asked to describe how their provider responded to their health concern, participants regularly referred to a provider taking time to listen, demonstrating genuine concern, and being accessible when needed. These relate to the "being attentive" dimension of the theme. When a provider takes the time to listen, an increased satisfaction with care among veteran patients has been observed (Phillips et al., 2017; Shamaskin-Garroway et al., 2018; Street et al., 2019; Trentalage et al., 2016). Also, when a veteran perceives a provider is demonstrating genuine concern, veterans feel more comfortable disclosing complicated health concerns (Cucciare et al., 2015; Ganzini et al., 2013; Jeffreys et al., 2010). Further, when a provider is perceived by a female veteran as being available when needed, a sense of value or worth is conveyed (Koblinsky et al., 2017).

One of the unique dimensions of the theme of affective support was "knowing me as a person by hearing my story". Participants described their military service as a part of their person; a part of their identity. This is not unexpected. From basic training and beyond, military persons go through an "indoctrination" of a particular branch's identified values and mission. They are governed by their superiors and must follow a very specific set of rules - on and off duty. The culture and language of the military becomes ingrained in the veteran (Goldenberg, Hamaoka, Santiago, \& McCarrol, n.d.; Meyer, 2015). The participants' military story was a part of who they are as a person. If a provider failed to hear the story, it was perceived as a threat to the participant's dignity. Providers who demonstrate a personal interest and who listen attentively convey worth to a female veteran (Koblinsky et al.,2017). In addition, the 
aforementioned provider behaviors are associated with care seeking and satisfaction (Chase et al., 2016; Ganzini et al., 2013).

Professional-technical competence. As noted earlier, the theme of recognizing and acknowledging women are veterans is unique to this population. A perceived unintentional gender discrimination was described by participants. It was related to their service roles and experiences not being fully understood, or at times downplayed, and the perceived lack of being screened because they were women. One participant commented, "it's a boy's world".

Gender discrimination has been described by other women veterans. Some have described more intentional discrimination, the forms varying from intimidation to harassment to denial of career advancement due to their gender, being perpetuated by their military male counterparts and/or superiors (Evans, Glover, Washington, \& Hamilton, 2018; Ingelse \& Messecar, 2015). It has been further perpetuated from within the VHA by a "male oriented" VHA system that is perceived as unreceptive to the needs of women veterans (Kotzias et al., 2018). Women in this study alluded to experiences (with both civilians and non-VHA, civilian providers) of being misidentified as the veteran's wife. Being misidentified as a male veteran's wife or daughter is not an uncommon experience (Koblinsky et al., 2017). Recognizing and acknowledging women who may have served promotes a sense of value or worthiness to women veterans who may perceive themselves as “...less 'deserving' of care compared to other veterans" (Koblinsky et al., 2017. p.126). Finally, it is important to recognize (or screen women for a history of military service) because some women veterans may not know to provide the history, don't self-identify as a veteran, or are embarrassed to seek help (Evans et al., 2018).

The theme of comprehending the military experience came from participants descriptions about providers not understanding what women veterans might have been exposed to while 
serving and the subsequent health consequences. It is important to note that some participants described experiences with providers who had a personal history of military service or more knowledge about the military, which they attributed to an increased understanding of their military role and experiences. This is likely associated with the proximity of a healthcare provider's location to a military base or areas of the country more populated by military personnel or veterans. For instance, participants who lived in Virginia or Florida noted that they lived near a large military base and that many providers in the area had previously served or had more exposure to persons who had served. Whereas participants living in West Virginia and Pennsylvania noted that providers in that area had limited exposure to military persons, limiting providers' understanding. This inference has been observed in the literature as well. Non-VHA, civilian providers perceived personal readiness to care for veteran populations has been shown to be increased for providers who have served or who have family members who have served (Kilpatrick et al., 2011; Miller et al., 2014).

The dimension of 'being thorough' in the third theme was related to the importance of considering military experience in the evaluation process. This was of key importance to several participants whose providers did not link their symptoms to their military experiences. Other studies in non-VHA, civilian provider populations indicate that several lack recognition of the significance of a history of military service related to health (Maiocco et al., in press; Vest et al., 2018a).

The participant concerns are not unfounded because many symptoms, which are also common in the civilian population, may not be obviously linked to a military condition if the history is unknown. For instance, insomnia and chronic pain may be presenting symptoms of post-traumatic stress disorder (Cox, McIntyre, \& Olatunji, 2018; Outcalt, Ang, Wu, Sargent, Yu, 
Bair, 2014). Further, symptoms of headache, irritability, dizziness, or memory problems are common presenting symptoms for traumatic brain injury (TBI) (Olenick, Flowers, \& Diaz, 2015). Asking about service informs the clinical history and ultimately diagnostic reasoning. Without asking, symptoms may be dismissed as something else that is inaccurate.

A provider's familiarity with a veteran patient's chart, or repetitive interaction with the same provider (continuity), both of which eliminated the need to start from scratch or repeat themselves at every appointment, has been associated with increased satisfaction and increased engagement in treatment in veteran populations (Bastian et al., 2014; Chase et al., 2016; Cucciare et al., 2015; Laird et al., 2013; Street et al., 2019). This finding in the literature was not initially apparent to be related to professional-technical competence. However, in this study, both were related to the description of a provider's professional-technical competence. Repetitive interaction with the same provider and/or the provider being familiar with the participants' chart before the encounter supported a participant's confidence in the providers competence.

Information giving. The dimension of uncomplicated explanation in the theme of information giving was also noted in the literature. In several studies, satisfaction was increased with the use of understandable language and clear expectations (Shamaskin-Garroway et al., 2018; Street et al., 2019). In addition, the contribution of uncomplicated explanation in facilitating decision making has been observed. Thorough explanation contributed to participants' perception that a healthcare encounter was patient centered (Balbale et al., 2014). A patient-centered encounter resulted in the participant feeling empowered and contributed to a sense of responsibility by participants to participant in their own care.

In this study, the provision of resources was important to participants' ability to participate in decision making, not only during the visit but also for engaging in treatment and/or 
self-management. Similarly, both Etingen et al. (2016) and Phillips et al. (2017) identified a relationship between thorough communication about a health concern and increased treatment adherence and self-management.

Decisional control. As noted earlier, unique to the theme of decision making is the dimension of collaborating. Many participants referenced the development of a partnership which included asking about their goals, providing options, and supporting their choice. Many of the participants attributed their desire for participating in decision making to their lack of ability to do so while in the military. The importance of soliciting a veteran patient's input and respecting his or her choice is evident in the literature. Blonigan et al. (2014) found that when a provider asks about a patient's interests and goals, holds a belief that patients can make their own choices, and respects those choices, veteran participants expressed higher levels of satisfaction. While the participants in this study indicated that decisional control was the most important factor in the patient-provider interaction and most participants desired a high level of control, this is not the case for all veterans. In two studies, veteran participants felt it was the responsibility of the provider to make decisions (Eliacin et al., 2015; Lederer et al., 2015). In both studies, provider expertise was cited as a contributing factor to participants' reluctance to participate in decision making. This perception was disputed by a participant in this study who stated, 'I don't think that just because they're a doctor means that they know always what's best for me".

\section{Critique of the Findings}

The trustworthiness of the findings of any scientific inquiry are not implicit. It is the duty of the researcher to ensure trustworthiness by demonstrating rigor. The rigor of qualitative inquiry is judged by the criteria of credibility, transferability, dependability, and confirmability 
(Shenton, 2004). The researcher's attention to the aforementioned criteria were described in Chapter 3. However, this study is not without limitations.

The participants in this study were asked to describe their interaction with a non-VHA, civilian provider. While some participants shared highlights, or brief examples of experiences with other providers, the main focus of their description revolved around interaction with one provider. Thus, the results of this study may not be representative of all interactions or encounters with non-VHA, civilian providers experienced by the participants.

Recruitment for this population was challenging. Despite multiple recruitment avenues, the primary pathway to participation (greater than $50 \%$ of participants) was via word of mouth by participants who referred their veteran peers. As a result, it is possible that the results may be biased due to the potential that participants referred peers with similar experiences. In addition, it may be that while broad, the recruitment efforts did not reach all eligible women veterans who might have been agreeable to participation, and their experiences have gone unheard.

The method of directed content analysis has an inherent tendency toward bias (Hsieh and Shannon, 2005). Use of pre-determined categories has the potential to limit the identification of new categories. To reduce bias, a second researcher, experienced in directed content analysis, performed a parallel analysis of the data. Both researchers identified that all the specific descriptors fit well in the pre-determined categories and no new categories were identified. To further demonstrate confirmability, an abbreviated audit trail is offered in the form of a table presenting the themes and associated condensed meaning units, and through the inclusion of specific descriptor exemplars within the text.

The sample size for this study was small at 13 participants and the inclusion criteria resulted in a more narrowly defined woman veteran population. In addition, while the sample 
represented most military branches, none of the participants had served in the Marines. The sample was also ethnically homogenous (>80\% Caucasian). Since racial/ethnic disparity is known to exist for women veterans (Carter et al., 2016; Lehavot, Beckman, Chen, Simpson, \& Williams, 2019), it is an important consideration with regard to transferability. Further, while the sample has been thoroughly described to enable other researchers and practitioners to identify the applicability of the study findings, the transferability to all women veterans who seek care from within the non-VHA, civilian sector is limited. For instance, the findings may not apply to women veterans who served in earlier war eras, women veterans who are dual users (concurrent use of non-VHA, civilian and VHA healthcare systems), or women who are still serving on active duty.

\section{Relevance to the Discipline of Nursing}

Substantive contribution to any discipline's body of knowledge is achieved by addressing the discipline's focus of concern. The central unifying focus for knowledge development in nursing has been defined as “...facilitating humanization, meaning, choice, quality of life, and healing in living and dying” (Willis, Grace, \& Roy, 2008, p. 32-33) for persons, families, and communities. This “...core epistemological structure can and should scaffold" (Thorne, 2016, p. 159) nursing inquiry. Further, maintaining nursing's disciplinary focus in the theory-research-practice triad is essential to prevent fragmentation of the discipline (Grace, Willis, Roy, \& Jones, 2016). In addition, articulating a research inquiry related to nursing's disciplinary focus results in knowledge development "...that is useful for nurses in practice and for nurses to contribute to multidisciplinary changes in health care" (Grace et al., 2016, p. 65). 
Nursing's “philosophic and practice dimensions...generate open metanarratives for scientific inquiry" (Reed, 1995, p.81). This inquiry about women veterans' experience with nonVHA, civilian healthcare providers is related to nursing practice and nursing's disciplinary focus and was guided by a nursing theoretical framework. Nursing theoretical frameworks provide weight and breadth to the structure of nursing research. Research findings strengthen the evidence base for nursing practice and provide support for the development or revision of nursing theory.

The IMCHB (Cox, 1982; 2003) theoretically conceptualizes the content and process of care, delivered to the patient as an individual, and the subsequent outcomes related to that care. The content and process of care is described as the patient-provider interaction element in the model. The findings of the study are organized by the patient-provider interaction factors in the model, professional-technical competence, information giving, decisional control, and affective support, and all relate back to nursing's central focus. As women veteran patients interact with a civilian provider, they are seeking “...healing in living and dying” (Willis et al., 2008). Understanding their experiences will help guide nurses, as well as other healthcare providers, to better facilitate humanization, meaning, choice, and quality of life. The thematic findings of knowing me as a person by hearing my story and being attentive to my concerns and recognizing and acknowledging women are veterans are about facilitating humanization and meaning for women veterans. The findings of uncomplicated explanation that facilitates decision making and collaborating by seeking input, providing options, and supporting decisions are about facilitating choice for women veterans. Finally, the findings of being thorough and accepting accountability for attending to a health concern and comprehending the military experience are about facilitating healing. 


\section{Implications for Policy, Research, and Practice}

The thematic findings of the study about women veterans' descriptions of their experience with civilian healthcare providers have implications for policy, research, and practice. Potential implications for each of these areas are offered below.

Policy. As noted in Chapter 1, the VHA Maintaining Systems and Strengthening Integrated Outside Networks or MISSION Act was implemented in 2018 (CRS, 2018). The act will enable women veterans who are eligible for VHA care to more easily access care from within the community or civilian sector. Thus, in addition to women veterans who are already obtaining care from within the civilian sector, more women veterans, many of whom have a service-connected disability, will be presenting to non-VHA, civilian healthcare facilities. Critiques of the prior policy, the Choice Act of 2014, which was a temporary act increasing access to civilian delivered healthcare, caution about the lack of consideration given to the development of community provider networks (Mattocks, Mengeling, Sadler, Baldor, \& Bastian, 2017). Requirements for non-VHA, civilian providers to receive education on military related healthcare issues are stipulated in the MISSION Act, but details concerning how the education will occur and be documented are absent.

While there has been a federal campaign, Joining Forces (White House, 2018) and a professional organization initiative, Have You Every Served in the Military (AAN, 2013), to increase provider attention and awareness about a patient's military history and veteran wellness, there are no mandates requiring inquiry about military status. Further only one state, West Virginia, mandates that health care providers (nurses, advance practice nurses, licensed practical nurses, psychologists, chiropractors, social workers and counselors) engage in two hours of continuing education annually on mental health conditions of veterans and their families (West 
Virginia Legislation, 2014). Despite these efforts, few community providers screen for military service, many express concerns regarding their personal competence to care for military personnel, and lack recognition of the significance of a history of military service (Maiocco, et al., 2018; Maiocco et al., in press; Vest et al., 2018a; Vest et al., 2018). The findings of this study, considered in the context of what is evident in the literature, can inform future policy development at the federal, state, and institutional level regarding implementation of mandatory screening for military status and the need for veteran-centric competency for continued practice. In addition, in the context of current literature and noted study limitations, the findings of this study also shed light on the need for additional education regarding the role, experience, and value of women veterans.

Research. The described patient-provider factors of the IMCHB were validated in the sample and no additional factors were identified, increasing the utility of the model for future research. While the findings of this study inform the use of the IMCHB in similar populations, additional descriptive studies evaluating the IMCHB are warranted to validate or extend the theory in other populations of women veterans. This includes, but is not limited to, women veterans from other war eras, women veterans obtaining care from the VHA or those who are dual users, and women who have not yet separated from the military. Further, samples that represent diverse ethnic backgrounds should be sought.

In this study, participants were not asked to disclose the nature of their health concern, extensive history of their military experiences, or their medical history; though several attributed their health concern to military service. Thus, it is not known whether these findings are similar for women veterans who have experienced a military-related health complication, versus those who have not. Further research in populations of women veterans who are known to have a 
diagnosed military-related complication, such as one of the "invisible wounds" or MST, are necessary. For instance, how do women veterans with a history of MST describe the patientprovider experience with both male and female non-VHA, civilian providers?

To this author's knowledge, this study is the first in a population of women veterans who served during the Post-9/11 war era, who exclusively obtain care from non-VHA, civilian providers. While it contributes to the body of knowledge about women veterans, there remains a disproportionate representation of women veterans in the literature. This includes research about women veterans who access either VHA or non-VHA, civilian healthcare sectors, or both. Additional research is needed to close the gap in knowledge about women veterans.

Recruitment. Data saturation was achieved after thirteen participant interviews. However, the time required to recruit such a small sample, a period of 16 months (average less than 1 participant per month), was quite lengthy. Further, despite multiple recruitment avenues (recruitment letters postal mailed to veterans accessing a non-VHA, civilian healthcare center, recruitment emails, recruitment flyers posted at local healthcare facilities and retail stores, and social media posts), the primary pathway to participation (greater than $50 \%$ of participants) was via word of mouth by participants who referred their veteran peers. Thus, this study experience indicates that recruitment from this population may be challenging. It must be noted that future studies requiring a larger number of participants may need a more innovative strategy for recruitment, such as community based participatory research (Israel, Schulz, Parker, \& Becker, 2001).

Practice. Current literature demonstrates that screening for veteran status is rarely employed in non-VHA, civilian healthcare settings. In addition, women veterans' perception of not being valued or recognized is evident in the literature. The findings of this study seem to 
suggest that this perception is re-enforced for some women veterans when providers fail to ask them if they have served. Further, by not asking, information vital to formulating a diagnosis or plan of care may be missed. Non-VHA, civilian providers should attend to collecting a thorough history, which includes inquiring about whether or not a patient has served in the military, whether they are male or female — all patients should be asked.

Moving forward, the IMCHB, informed by the study findings, may be used to develop interventions to enhance care delivered by non-VHA, civilian providers. The themes highlight areas of importance for each factor in the patient-provider element, a provider's affective response, professional technical competence, sharing of decisional control, and information giving, when working with women veterans who are similar to the description of the sample in this study. For instance, an intervention to enhance a provider's professional-technical competence might include education about the roles of women veterans who served during the post-9/11 war era, the various exposures potentially encountered in areas to which they might have been deployed, and the subsequent, potential health complications including, screening, assessment, and evidence-based treatment of such.

\section{Summary}

The findings of this study are an important contribution to the existing literature about non-VHA, civilian delivered healthcare. The themes that emerged from the study provide rich descriptions of women veterans' interactions with non-VHA, civilian providers. The themes indicate an absence of consistent screening of female patients, a deficit in knowledge regarding the role and experiences of women during military service, and the need for additional training about military-related health conditions. Further, the themes highlight the importance of the woman veteran's story, as well as the need to recognize and acknowledge the service of women 
veterans. Finally, the themes inform the use of the IMCHB to guide research, practice, and policy related to the care of women veterans who obtain care from non-VHA, civilian providers. 


\section{References}

Abraham, T., Wrights, P., White, P. Booth, B., \& Cucciare, M. (2017). Feasibility and acceptability of shared decision-making to promote alcohol behavior change among women veterans: Results from focus groups. Journal of Addictive Diseases, 36(4), 252263. doi: 10.1080/10550887.2017.13733318

American Academy of Nursing. (n.d.) Have you served in the military? Retrieved from http://www.haveyoueverserved.com/

Atkins, D. (2013). Health services research on women veterans: A critical partner on the road to patient-centered care. Journal of General Internal Medicine, 28(2), s498-499. doi: $10.1007 / \mathrm{s} 11606-013-2472-7$

Balbale, S., Morris, M., \& LaVela, S. (2014). Using photovoice to explore patient percetions of patient-centered care in the veterans affairs health care system. Patient, 7, 187-195. doi: $10.1007 / \mathrm{s} 40271-014-0044-5$

Bagalman, E. (2013). Mental disorder among OEF/OIF veterans using VA health care: facts and figures. Retrieved from the Congressional Research Service website: https://www.fas.org/sgp/crs/misc/R41921.pdf

Bastian, L., Trentalange, M., Murphy, T., Brandt, C., Bean-Mayberry, B., Maisel, N., ...Haskell, S. (2014). Association between women veterans' experience with VA outpatient health care and designation as a woman's health provider in primary care clinics. Women's Health Issues, 24(6), 605-612. doi: 10.1016/j.whi.2014.07.005

Bastian, L., Mattocks, K., Rosen, A., Hamilton, A., Bean-Mayberry, B., ...Yano, E. (2015). Informing policy to deliver comprehensive care for women veterans. Medical Care, 53(4supp), s1-s4. doi: 10.1097/MLR.0000000000000344 
Batuman, F., Bean-Mayberry, B., Goldzweig, C., Huang, C., Miake-Lye, I., Washington, D.,...Shekelle, P. (2011). Health effects of military service on women veterans. VA-ESP Project \# 05-226. Retrieved from https://www.hsrd.research.va.gov/publications/esp/women-vets-EXEC.pdf

Bergman, A., Frankel, R., Hamilton, A., \& Yano, E. (2015). Challenges with delivering genderspecific and comprehensive primary care to women veterans. Women's Health Issues, 25(1), 28-34. doi: 10.1016/j.whi.2014.10.004

Blonigen, D., Bui, L., Harris, A., Hepner, K., \& Kivlahan, D., (2014). Perceptions of behavioral health care among veterans with substance use disorders: Results from a national evaluation of mental health services in the Veterans Health Administration. Journal of Substance Abuse Treatment, 47, 122-129. doi: 10.1016/j.jsat.2014.03.005

Bohnert, A., Zivin, K., Welsh, D., \& Kilbourne, A. (2011). Ratings of patient-provider communication among veterans: Serious mental illnesses, substance use disorders, and the moderating role of trust. Health Communication, 26, 267-274. Doi: $10.1080 / 10410236.2010 .549813$

Boyd, M., Bradshaw, W., \& Robinson, M. (2013). Mental health issues of women deployed to Iraq and Afghanistan. Archives of Psychiatric Nursing, 27(1), 10-22. doi:10.1016/j.apnu.2012.10.005

Brown, S.J. (1992). Tailoring nursing care to the individual client: empirical challenge of a theoretical concept. Research in Nursing \& Health, 15(1), 39-46.

Carlson, B., Stromwall, L., \& Lietz, C. (2013). Mental health issues in recently returning women veterans: Implications for practice. Social Work, 58(2), 105-114. doi:10.1093/sw/swt001 
Carter, K. (1997). Correlates of health behaviors of community living older adults (Doctoral dissertation). Retrieved from https://search-proquestcom.www.libproxy.wvu.edu/pqdtglobal/index?accountid=2837

Carter, K. \& Kulbok, P. (1995). Evaluation of the interaction model of client health behavior through the first decade of research. Advances in Nursing Science, 18(1), 62-73.

Carter, A., Borrero, S., Wessel, C., Washington, D., Bean-Mayberrry, ...Zickmund, S., (2016). Racial and ethnic health care disparities among women in the veterans affairs healthcare system: A systematic review. Women's Health Issues, 26(4), 401-409. doi:

10.1016/j.whi.2016.03.009 https://doi.org/10.1016/j.whi.2016.03.009

Center for Deployment Psychology. (2014). Military culture: Core competencies for health care professionals. Retrieved from Center for Deployment Psychology website: http://deploymentpsych.org/military-culture-course-modules

Chase, R., McMahon, S., \& Winch, P. (2016). “Tell me what you don't remember”: Careseeking facilitators and barriers in the decade following repetitive blast exposure among army combat veterans. Military Medicine, 181(2), 116-122. doi: 0.7205/MILMED-D-1400471

Cleary, M., Horsfall,J., \& Hayter, M. (2014). Data collection and sampling in qualitative research: does size matter? Journal of Advanced Nursing, 70(3), 473-475. doi: 10.1111/jan.12163

Collingridge, D. \& Gantt, E. (2008). The quality of qualitative research. American Journal of Medical Quality, 23(5), 389-395. doi: 10.1177/1062860608320646 
Congressional Research Service. (2018). VA Maintaining Internal Systems and Strengthening Integrated Outside Networks Act of 2018 (VA MISSION Act; P.L.115-182). Retrieved at https://fas.org/sgp/crs/misc/R45390.pdf

Conrad, P \& Armstrong, M. (2016). Advocating for deployed women veterans' health differences, difficulties, and disparities. Nursing Forum, 51(4), 225-232. doi: $10.1111 /$ nuf. 12143

Convoy, S. \& Westphal, R. (2013). The importance of developing military cultural competence. Journal of Emergency Nursing Online, 39(6), 591-594. doi: 10.1016/j.jen.2013.08.010

Cordasco, K., Mengeling, M., Yano, E. \& Washington, D. (2016). Health and health care access of rural women veterans: findings from the national survey of women veterans. Journal of Rural Health, 32(4), 397-406. doi: 10.1111/jrh.12197

Cox, C. (1982). An interaction model of client health behavior: theoretical prescription for nursing. Advances in Nursing Science, 5(10), 41-56.

Cox, C. (1984). The individual as a client. In J. Sullivan (Ed), Direction for community health nursing (pp. 129-172). Boston: Blackwell Scientific

Cox, C. (2003). A model of health behavior to guide studies of childhood cancer survivors. Oncology Nursing Forum, 30(5), e92-e99. Doi:10.1188/03.ONF.E92-E99

Cox, C. \& Roghmann, K. (1984). Empirical test of the interaction model of client health behavior. Research in Nursing \& Health, 7(4), 275-285. doi: 10.1002/nur.4770070406

Cox, R., McIntyre, A. \& Olatunji, B. (2018). Interactive effects of insomnia symptoms and trauma exposure on PTSD: Examination of symptom specificity. Psychological Trauma, Theory, Research, Practice, and Policy, 10(5), 508-514. doi: 10.1037/tra0000336 
Creech, S., Pulverman, C., Crawford, J., Holliday, R., Monteith, L., Lehavot, K., Olson-Madden, J., \& Kelly, U. (2019). Clinical complexity in women veterans: a systematic review of the recent evidence on mental health and physical health comorbidities. Behavioral Medicine, 1-16. Doi:10.1080/08964289.2019.1644283

Cucciare, M., Lewis, E., Hoggatt, K., Bean-Mayberry, B., Timko, C., Durazo, E., ...Frayne, S. (2015). Factors affecting women's disclosure of alcohol misues in primary care: A qualitative study with U.S. military veterans. Women's Health Issues, $x x x-x x$, 1-8. doi: 10.1016/j.whi.2015.07.010

Curtis, S., Gesler, W., Smith, G., \& Washburn, S. (2000). Approaches to sampling and case selection in qualitative research: examples in the geography of health. Social Science \& Medicine, 50, 1001-1014.

Danan, E., Krebs, E., Ensrud, K., Koeller, E., MacDonald, R., Velasquez, T., Greer, N., \& Wilt, T. (2017). An evidence map of the women veterans' health research literature (20082015). Journal of Internal Medicine, 32(12), 1359-1376. doi: 10.1007/s11606-017-41525

DAV. (2018). Women veterans: The journey ahead. Retrieved at https://www.dav.org/wpcontent/uploads/2018_Women-Veterans-Report-Sequel.pdf

Department of Health, Education, and Welfare. (1979). The Belmont Report: Ethical principles and guidelines for the protection of human subjects of research. Retrieved at http://www.hhs.gov/ohrp/regulations-and-policy/belmont-report/

Dodds, C. \& Kiernan, M. (2019). Hidden veterans: A review of the literature on women veterans in contemporary society. Illness, Crisis \& Loss, 24(4), 293-310. doi:

$10.1177 / 1054137319834775$ 
Elacin, J., Salyers, M., Kukla, M., \& Matthias, M. (2015). Factors influencing patients’ preferences and perceived involvement in shared decision-making in mental health care. Journal of Mental Health, 24(1), 24-28. doi: 10.3109/09638237.2014.954695

Elo, S. \& Kyngas, H. (2007). The qualitative content analysis process. Journal of Advanced Nursing, 62(1), 107-115. Doi: 10.1111/j.1365-2648.2007.04569.x

Elo, S., Kaariainen, M., Kanste, O., Polkki, T., Utriainen, K., \& Kyngas, H. (2014). Qualitative content analysis: A focus on trustworthiness. SAGE Open, 1-10. Doi: $10.1177 / 2158244014522633$

Etingen, B., Miskevics, S., LaVella, S. (2016). Assessing the association of patient-reported perceptions of patient-centered care as supplemental measures of health care quality in VA. Journal of General Internal Medicine, 31(1), S10-20. doi: 10.1007/s11606-015$3557-2$

Evans, E., Glover, D., Washington, D., \& Hamilton, A. (2018). Psychosocial factors that shape substance abuse and related mental health of women military veterans who use community-based services. Substance Use \& Misuse, 53(11), 1878-1892. doi: $10.1080 / 10826084.2018 .1441309$

Fischer, E., McSweeney, J., Wright, P., Cheney, A., Gurran, G., Henderson, K., \& Fortney, J. (2016). Overcoming barriers to sustained engagement in mental health care: Perspectives of rural veterans and providers. The Journal of Rural Health, 32, 429-438. doi: 10.1111/jrh.12203

Flannery, M. (2016). Common perspectives in qualitative research. Oncology Nursing Forum, 43(4), 517-518. doi: 10.1188/16.ONF.517-518 
Frayne, S.M., Phibbs, C.S., Saechao, F., Maisel, N.C., Friedman, S.A., Finlay A.,... Haskell, S. (2014). Sourcebook: Women Veterans in the Veterans Health Administration. Volume 3. Sociodemographics, Utilization, Costs of Care, and Health Profile. Women's Health Evaluation Initiative, Women's Health Services, Veterans Health Administration, Department of Veterans Affairs, Washington DC. Retrieved at http://www.womenshealth.va.gov/latestinformation/publications.asp

Frayne, S.M., Wei, Y., Yano, E., Anath, L., Iqbal, S., Thraikill, A., \& Phibbs, C. (2006). Gender and Use of Care: Planning for Tomorrow's Veterans Health Administration. Journal of Women's Health, 16(8), 1188-1199. doi: 10.1089/jwh.2006.0205

Fredricks, T. \& Nakazawa, M. (2015). Perceptions of physicians in civilian medical practice on veterans' issues related to health care. The Journal of the American Osteopathic Association, 115(6), 360-368. doi:10.7556/jaoa.2015.076

Ganzini, L, Denneson, L., Press, N., Bair, M., Helmer, D., Poat, J., \& Dobscha, S. (2013). Trust is the basis for effective suicide risk screening and assessment in veterans. Journal of General Internal Medicine, 28(9), 1215-1221. doi: 10.1007/s11606-013-2412-6

Garvin, J.T. (2012). A tailored intervention program for overweight and obese veterans: Who benefits and when (Doctoral dissertation). Retrieved from https://search-proquestcom.www.libproxy.wvu.edu/pqdtglobal/index $?$ accountid=2837

Goldenberg, M., Hamaoka, D., Santiago, P., \& McCarroll, J. (n.d.). Basic training: A primer on military life and culture for health care providers. F. Edward Hebert School of Medicine, Uniformed Service University of the Health Sciences. Retrieved at http://deploymentpsych.org/sites/default/files/mc_resources/Basic\%20Training\%20A\%2 0primer\%20on\%20military\%20life\%20and\%20culture.pdf 
Grace, P., Willis, D., Roy, C., \& Jones, D. (2016). Profession at the crossroads: A dialog concerning the preparation of nursing scholars and leaders. Nursing Outlook, 64(1), 6170. doi: 10.1016/j.outlook.2015.10.002

Graneheim, U. \& Lundman, B. (2004). Qualitative content analysis in nursing research: concepts, procedures and measures to achieve trustworthiness. Nurse Education Today, 24, 105-112. Doi: 10.1016/j.nedt.2003.10.001

Hinojosa, R.,Hinojosa, S., Nelson, K., \& Nelson, D. (2010). Veteran family reintegration, primary care needs, and the benefit of the patient-centered medical home model. The Journal of the American Board of Family Medicine, 23(6), 770-774. doi: 10.3122/jabfm.2010.06.100094

Hoffmire, C., Kemp, J., \& Bossarte, R. (2015). Changes in suicide mortality for veterans and nonveterans by gender and history of VHS service use, 2000-2010. Psychiatric Services, 66(9), 959-965. Doi:10.1176/appi.ps.201400031

Hoglund, M. \& Schwartz, R. (2014). Mental health in deployed and nondeployed veteran men and women in comparison with their civilian counterparts. Military Medicine, 179(1), 1925. doi: 10.7205/MILMED-D-13-00235

Hsieh, H. \& Shannon, S. (2005). Three approaches to qualitative content analysis. Qualitative Health Research, 15(9). 1277-1288. Doi: 10.1177/1049732305276687

Ingelse, K. \& Messecar, D. (2016). Rural women veterans' use and perception f mental health services. Archives of Psychiatric Nursing, 30, 244-248. doi: 10.1016/j.apnu.2015.11.008

IOM. (2013). Report brief: Returning home from Iraq and Afghanistan: Assessment of readjustment needs of veterans, service members, and their families. Retrieved from IOM 
website: http://www.iom.edu/Reports/2013/Returning-Home-from-Iraq-and-

\section{$\underline{\text { Afghanistan.aspx }}$}

Israel, B., Schulz, A., Parker, E., \& Becker, A. (2001). Community-based participatory research recommendations for promoting a partnership approach in health research. Education for Health, 14(2), 182-197. Doi: 10.1080/13576280110051055

Jackonis, M., Deyton, L., \& Hess, W. (2008). War, its aftermath, and U.S. health policy: toward a comprehensive health care program for America's military personnel, veterans, and their families. Journal of Law, Medicine, \& Ethics, 36(4), 677-689. doi:10.1111/j.1748-1720X.2008.00322.x

Jeffreys, M., Leibowitz, R., Finley, E., \& Arar, N. (2010). Trauma disclosure to health care professionals by veterans: clinical implications. Military Medicine, 175(10), 719-724.

Katon, J \& Reiber, G. (2013). Major traumatic limb loss among women veterans and service members. Journal of Rehabilitation Research \& Development, 50(2), 173-182

Kilpatrick, D., Best, C., Smith, D., Kudler, H., \& Cornelison-Grant, V. (2011). Serving those who have served: education needs of health care providers working with military members, veterans, and their families. Charleston, SC: Medical University of South Carolina Department of Psychiatry, National Crime Victims Research \& Treatment Center. Retrieved from http://deploymentpsych.org/sites/default/files/mc_resources/Serving\%20Those\%20Who \%20Have\%20Served.pdf

Kimerling, R., Pavao, J. Valdez, C., Mark, H., Hyun, J., \& Saweikis, M. (2011). Military sexual trauma and patient perceptions of veteran health administration health care quality. Women's Health Issues, 21(4S), S145-S151. doi: 10.1016/j.whi.2011.04.007 
Koblinsky, S., Leslie, L., \& Cook, E. (2014). Treating behavioral health conditions of OEF/OIF veterans and their families: a state needs assessment of civilian providers. Military Behavioral Health, 2, 162-172. doi:10.1080/21635781.2014.890884

Koblinsky, S., Schroeder, A., \& Leslie, L. (2017). “Give us respect, support and understanding”: Women veterans of Iraq and Afghanistan recommend strategies for improving their mental health. Social Work in Mental Health, 15(2), 121-142. doi:

$10.1080 / 15332985.2016 .1186134$

Koenig, C., Maguen, S., Monroy, J., Mayott, L, \& Seal, K. (2014). Facilitating culture-centered communication between health care providers and veterans transitioning from military deployment to civilian life. Patient Education and Counseling, 95, 414-420. doi:10.1016/j.pec.2014.03.016

Kotzias, V., Engel, C., Ramchand, R., Ayer, L., Predmore, Z., Ebener, P., ...Karras, E. (2018). Mental health service preferences and utilization among women veterans in crisis: Perspective of veterans crisis line responders. Journal of Behavioral Health Services \& Research, 29-42. doi: 10.1007/s11414-018-9635-6

Laird, J.E., Tolentino, J.C., \& Gray, C. (2013). Patient greeting preferences for themselves and their providers in a military family medicine clinic. Military Medicine, 178(10), 11111114. doi: 10.7205/MILMED-D-12-00360

Lee, T.T., Westrup, D., Ruzek, J., Keller, J., \& Weitlauf, J. (2007). Impact of clinician gender on examination anxiety among female veterans with sexual trauma: A pilot study. Journal of Women's Health, 16(9), 1291-1299. doi: 10.1089/jwh.2006.0208 
Lederer, S., Fischer, M., Gordon, H., Wadhwa, A., Popli, S., \& Gordon, E. (2015). Barriers to effective communication between veterans with chronic kidney disease and their healthcare providers. Clinical Kidney Journal, 8(6), 766-771. doi: 10.1019/ckj/sfv079

Lehavot, K., Beckman, K. L., Chen, J. A., Simpson, T. L., \& Williams, E. C. (2019). Race/ethnicity and sexual orientation disparities in mental health, sexism, and social support among women veterans. Psychology of Sexual Orientation and Gender Diversity, 6(3), 347-358. doi: 10.1037/sgd0000333

Lehavot, K., Der-Martisrosian, C., Simpson, T., Sadler, A., \& Washington, D. (2013). Barriers to care for women veterans with posttraumatic stress disorder and depressive symptoms. Psychological Services, 10(2), 203-212. doi: 10.1037/a0031596

Lahavot, K., Hoerste, K., Nelson,K., Jakupcak, M., \& Simpson, T. (2012). Health indicators for military, veteran, and civilian women. American Journal of Preventative Medicine, 42(5), 473-480. doi: 10.1016/j.amepre.2012.01.006

Levander, X. \& Overland, M. (2015). Care of women veterans. Medical Clinics of North America, 99(3), 651-662. doi:10.1016/j.mena.2015.01.013

Lincoln, Y.S. \& Guba, E.G. (1985). Naturalistic inquiry. Newbury Park, CA: Sage

Maguen, S., Cohen, B., Ren, L., Bosch, J., Kimerling, R. \& Seal, K. (2012). Gender differences in military sexual trauma and mental health diagnoses among Iraq and Afghanistan veterans with posttraumatic stress disorder. Women's Health Issues, 22(10), 61-66. doi: 10.1016/j.whi.2011.07.010

Maiocco, G., Stroupe, L., Rhoades, A., \& Vance, B. (2018). Care of veterans in a non-veteran health administration hospital: What is the status of nursing practice after continuing education? Journal of Clinical Nursing, 00,1-8. doi: 10.1111/jocn.14641 
Maiocco, G., Vance, B., \& Dicchiacchio, T. (in press). Readiness of non-Veterans Health Administration advanced practice registered nurses (APRN) to care for those who have served: A multi-method descriptive study. Policy, Politics, \& Nursing Practice, 1-26.

Maisel, N., Haskell, S., Haynes, P., Balasubramanian, B., Torgal, A., Ananth, L., ... Fraynes, S. (2015). Readying the workforce: Evaluation of VHA's comprehensive women's health primary care provider initiative. Medical Care, 53(4), 39-46. Doi:

Mattocks, K. (2015). Care coordination for women veterans: Bridging the gap between systems of care. Medical Care, 53(4supp), s8-s9. doi:10.1097/MLR.0000000000000339

Mattocks, K.M., Mengeling, M., Sadler, A., Baldor, R., \& Bastian, L. (2017). The veterans choice act: A qualitative examination of rapid policy implementation in the department of veterans affairs. Medical Care, 55, S71-S75. doi: 10.1097/mlr.0000000000000667

Meredith, L., Wang, Y., Okunogbe, A., Bergman, A., Canelo, I., Darling, J., \& Yano, E. (2017). Attitudes, practices, and experiences with implementing a patient-centered medical home for women veterans. Women's Health Issues, 27(2), 221-227. doi:

10.1016/j.whi.2016.11.008

Meyer, E. (2015). The importance of understanding military culture. Academic Psychiatry, 39, 416-418. doi: 10.1007/s40596-015-0285-1

Meyer, E., Writer, B., \& Brim, W. (2016). The importance of military cultural competence. Current Psychiatry Report, 18 (26), 1-9. doi: 10.1007/s11920-016-0662-9

Miller, K., Finn, J., \& Newman, E. (2014). Are communities ready? Assessing providers' practices, attitudes, and knowledge about military personnel. Professional Psychology: Research and Practice, 45(6), 398-404. doi:10.1037/a0036774 
Miller, E. \& Intrator, O. (2012). Veterans use of non-VHA services: Implications for policy and planning. Social Work in Public Health, 27, 379-39. doi:10.1080/19371910903176197

Mohler, K. \& Sankey-Deemer, C. (2017). Primary care providers and screening for military service and PTSD. American Journal of Nursing, 117(11), 22-28. doi:

10.1097/01.NAJ.0000526720.34516.4b

NCVAS (2016). West Virginia. Retrieved from https://www.va.gov/vetdata/docs/SpecialReports/State_Summaries_West_Virginia.pdf

NCVAS. (2016). Profile of Women Veterans: 2015. Retrieved from https://www.va.gov/vetdata/docs/specialreports/women_veterans_profile_12_22_2016.pd $\underline{\mathrm{f}}$

NCVAS. (2017). Women veteran report: 2015. Retrieved from https://www.va.gov/vetdata/docs/SpecialReports/Women_Veterans_2015_Final.pdf

Olenick, M., Flowers, M., \& Diaz, V. (2015). US veterans and their unique issues: enhancing health care professional awareness. Advances in Medical Education and Practice, 6, 635639. doi: 10.2147/AMEP.S89479

Outcalt, S., Ang, D., Wu, J., Sargent, C., Yu, Z., \& Bair, M. (2014). Pain experience in Iraq and Afghanistan veterans with comorbid chronic pain and posttraumatic stress. Journal of Rehabilitation and Research Development, 51(4), 559-570. doi:

10.1682/JRRD.2013.06.0134

Pandey, N., Ashfaq, S. N., Dauterive, E. W., 3rd, MacCarthy, A. A., \& Copeland, L. A. (2018). Military sexual trauma and obesity among women veterans. Journal of Women's Health, 27(3), 305-310. doi: 10.1089/jwh.2016.6105 
Pease, J., Billera, \& Gerard, G. (2015). Military culture and the transition to civilian life: Suicide risk and other considerations. Social Work, 61(1), 83-86.doi: 10.1093/sw/swv050

Petty, N., Thomson, O., \& Stew, G. (2012). Ready for a paradigm shift? Part 1: introducing the philosophy of qualitative research. Manual Therapy, 17, 267-274.

doi:10.1016/j.math.2012.03.006

Phillips, L., McAndrew, L., Laman-Maharg, B., \& Bloeser, K. (2017). Evaluating challenges for improving medically unexplained symptions in US military veterans via provider communication. Patient Education and Counseling, 100, 1580-1587. doi:

10.1016/j.pec.2017.03.011

Polit, D. \& Beck, C. (2012a). Theoretical frameworks. In D. Polit \& C. Beck (Eds.), Nursing research: Generating and assessing evidence for nursing practice. (pp. 126-149). Hong Kong, China: Lippincott, Williams, \& Wilkins.

Polit, D. \& Beck, C. (2012b). Introduction to nursing research in an evidence-based practice environment. In D. Polit \& C. Beck (Eds.), Nursing research: Generating and assessing evidence for nursing practice. (pp. 3-24). Hong Kong, China: Lippincott, Williams, \& Wilkins.

Reed, P. (1995). A treatise on nursing knowledge development for the $21^{\text {st }}$ century: beyond postmodernism. Advances in Nursing Science, 17(3), 70-84.

Resnick, E., Mallampalli, M., \& Carter, C. (2012). Current challenges in female veterans' health. Journal of Women's Health, 21(9), 895-900. doi: 10.1089/jwh.2012.3644

Rice, V., Fox, D., Lepczyk, M., Sieggreen, M., Mullin, M., Jarosz, P, \& Templin, T. (1994). A comparison of nursing interventions for smoking cessation in adults with cardiovascular health problems. Heart \& Lung, 23(6), 473-486. 
Rivera, J. \& Johnson, A. (2014). Female veterans of operations enduring and Iraqi freedom status and future directions. Military Medicine, 179(2), 133-136. doi: 10.7205/MILMEDD-13-00425

Rogers, R. G., Clark, E. A., Murata, E., Cichowski, S. B., Murata, A., \& Murata, G. (2017). Military sexual trauma in female veterans is associated with chronic pain conditions. Military Medicine, 182(9), e1895-e1899. doi: 10.7205/MILMED-D-1600393

Ross, P, Ravindranath, D., Clay, M., \& Lypson, M. (2015). A greater mission: Understanding military culture as a tool for serving those who have served. Journal of Graduate Medical Education, 519-522. doi: 10.4300/JGME-D-14-00568.1.

Rubin, H. \& Rubin I. (2012). Designing main questions and probes. In H. Rubin \& I Rubin (Eds.), Qualitative interviewing (2 ${ }^{\text {nd }}$ ed.) (pp 152-172). Thousand Oaks: SAGE Publications, Inc.

Runnals, J., Garovoy, N., McCutcheon,S., Robbins, A, Mann-Wrobel, M., \& Elliott, A. (2014). Systematic review of women veterans' mental health. Women's Health Issues, 24(5), 485-502. doi: 10.1016/j.whi.2014.06.012

Sandelowski, M. (1995). Focus on qualitative methods: Sample size in qualitative research. Research in Nursing \& Health, 18, 179-183.

Sandelowski, M. (2000). Whatever happened to qualitative description? Research in Nursing \& Health, 23, 234-340.

Sandelowski, M. (2010). What's in a name? Qualitative description revisited. Research in Nursing \& Health, 33, 77-84. doi: 10.1002./nur.20362 
Schreier, M. (2013). Qualitative content analysis. In U. Flick (Ed.), The SAGE handbook of qualitative data analysis. (pp. 170-183). London: SAGE Publications, Ltd.

Shamaskin-Garroway, A.M., Knobf, M., Adams, L., \& Haskell, S. (2018). "I think it’s pretty much the same, asit should be": Perspectives of inpatient care among women veterans. Qualitative Health Research, 28(4), 600-609. doi: 10.1177/1049732317746380

Shen, C. \& Sambamoorthi, U. (2012). Associations between health-related quality of life and financial barriers to care among women veterans and women non-veterans. Women \& Health, 51, 1-17. doi: 10.1080/03630242.2011.641713

Shenton, A.K. (2004). Strategies for ensuring trustworthiness in qualitative research projects. Education for Information, 22, 63-75.

Slatore, C.G., Feemster, L., Au, D., Englelberg, R., Curtis, J., Uman, J., \& Reinker, L. (2014). Which patient and clinician characteristics are associated with high-quality communication among veterans with chronic obstructive pulmonary disease? Journal of Health Communication, 19, 907-921. doi: 10.1080/10810730.2013.864732

Slatore, C.G., Golden, S., Ganzini, L., Wiener, R. \& Au, D. (2015). Distress and patient-centered communication among vetereans with incidental (not screen-detected) pulmonary nodules. Annals of the American Thoracic Society, 12(2), 184-192. doi: 10.1513/AnnalsATS.201406-2830C

Street, A., Shin, M., Marchany, K., McCaughey, V., Bell, M., \& Hamilton, A. (2019). Veterans' perspectives on military sexual trauma-related communication with VHA providers. Psychological Services, 1-11. Doi:10.1037/ser0000395

Strong, J., Crowe, B., \& Lawson, S. (2018). Female veterans: Navigating two identities. Clinical Social Work, 46, 92-99. Doi: 10.1007/s10615-017-0636-3 
Tanielian, T., Jaycox, L., Adamson, D., Burnam, M., Burns, R., Caldarone, L., ... Yochelson, M. (2008). Invisible wounds of war: Psychological and cognitive injuries, their consequences, and services to assist recovery. Santa Monica, CA: RAND Corporation. Retrieved from http://www.rand.org/pubs/monographs/MG720

Tanielian, T., Farris, C., Epley, C., Farmer, C., Robinson, E., Engel, C., . . Jaycox, L. (2014). Ready to Serve: Community-Based Provider Capacity to Deliver Culturally Competent, Quality Mental Health Care to Veterans and Their Families. RAND Corporation. Retrieved from http://www.jstor.org/stable/10.7249/j.ctt14bs1qk

The Whitehouse (n.d.) About joining forces, Retrieved at https://obamawhitehouse.archives.gov/joiningforces/about

Thorne, S. (2016). The status and use value of qualitative research findings: New ways to make sense of qualitative work. In Lipscomb (Ed.), Exploring evidence based practice: Debates and challenges in nursing (pp. 151-164). New York, NY: Routledge.

Trentalange, M., Bielawski, M., Murphy, T., Lessard, K., Brandt, C., Bean-Mayberry, B.,...Bastian, L. (2016). Patient perception of enough time spent with provider is a mechanism for improving women veterans' experiences with VA outpatient health care. Evaluation \& the Health Professions, 39(4), 460-474. doi: 10.1177/0163278716629523

Troumbley, P.F. (1988). A comparison of the health, risk, and physical fitness of overweight and normal weight soldiers (Doctoral dissertation). Retrieved from https://search-proquestcom.www.libproxy.wvu.edu/pqdtglobal/index?accountid=2837

Troumbley, P.F. \& Lenz, E.R. (1992). Application of Cox's interaction model of client health behavior in a weight control program for military personnel: a preintervention baseline. Advances in Nursing Science, 14(4): 65-78. 
U.S. Congress. (2014). Text of the Veterans Access, Choice, and Accountability Act of 2014. Retrieved from https://www.govtrack.us/congress/bills/113/hr3230/text

U.S. Department of Veterans Affairs. (2015). Study of barriers for women veterans to VA health care: Final report. Retrieved at https://www.womenshealth.va.gov/docs/Womens\%20Health\%20Services_Barriers\%20to \%20Care\%20Final\%20Report_April2015.pdf

U.S. Department of Veterans Affairs. (2017). Analysis of VA health care utilization among Operation Enduring Freedom (OEF), Operation Iraqi Freedom (OIF), and Operation New Dawn (OND) Veterans. Retrieved at https://www.publichealth.va.gov/docs/epidemiology/healthcare-utilization-reportfy2015-qtr3.pdf\#

Vaismoradi, M., Turunen, H., Bondas, T. (2013). Content analysis and thematic analysis: Implications for conducting a qualitative descriptive study. Nursing \& Health Sciences, 15(3):398-405. doi: 10.1111/nhs. 12048

Vance, B., Alhussain, A., \& Sambamoorthi, U., (2019). Five-year trend in healthcare access and patient-reported health outcomes among women veterans. Nursing Forum, 1-9. doi: $10.1111 /$ nuf. 12411

Vest, B., Kulak, J., Hall, V., \& Homish, G. (2018a). Addressing patients' veteran status: primary care providers' knowledge, comfort, and educational needs. Family Medicine, 50(6), 455459. doi: 10.22454/FamMed.2018.795504

Vest, B., Kulak, J., \& Homish, G. (2018). Caring for veterans in US civilian primary care: qualitative interviews with primary care providers. Family Practice, 36 (3), 343-350. doi:10.1093/fampra/cmy078 
Villagran, M., Ledford, C., \& Canzona, M. (2015). Women's health identities in the transition from military member to service veteran. Journal of Health Communication, 20, $1125-$ 1132. doi: $10.1080 / 10810730.2015 .1018619$

Vogt, D., Bergeron, A., Salgado, D., Daley, J., Ouimette, P., \& Wolfe, J. (2006). Barriers to Veterans Health Administration Care in a Nationally Representative Sample of Women Veterans. Journal of General Internal Medicine, 21(3), S19-25. doi:

$$
\text { 10.1111/j.1525-1497.2006.00370.x }
$$

Wagner, D., Bear, M., \& Davidson, N. (2011). Measuring patient satisfaction with postpartum teaching methods used by nurses within the interaction model of client health behavior. Research \& Theory for Nursing Practice, 25(3), 176-190. doi: 10.1891/15416577.25 .3 .176

Wands, L. (2013). "No one gets through it OK": The health challenge of coming home from war. Advances in Nursing Science, 36(3), 186-99. doi: 10.1097/ANS.Ob013e31829edcbe Washington, D., Bean-Mayberry, B., Riopelle, D., \& Yano, E. (2011). Access to care for women veterans: delayed healthcare and unmet needs. Journal of General Internal Medicine, 26, 655-661. doi: 10.1007/s11606-011-1772-x

Washington, D., Farmer, M., Mor, S., Canning, M., \& Yano, E. (2015). Assessment of the healthcare needs and barriers to VA use experienced by women veterans. Medical Care, 53(4 supp), s23-31. doi: 10.1097/MLR.0000000000000312

Weiss, E., Coll, J., \& Metal, M. (2011). The influence of military culture and veteran worldviews on mental health treatment: practice implications for combat veteran help-seeking and wellness. The International Journal of Health, Wellness, and Society, 1(2), 75-86. 
Wengraf, T. (2011). Lightly and heavily structured depth interviewing: Theory-questions and interviewer-questions. In Qualitative Research Interviewing. (pp 60-70). SAGE Publications, Ltd: London.

West Virginia Legislature. (2014). Article 1. General provisions applicable to all state boards of examination or registration referred to in chapter. \$30-1-7a. Continuing education. Retrieved at http://www.wvlegislature.gov/wvcode/chapterentire.cfm?chap=30\&art=1\&section=7a

Westphal, R. \& Convoy, S. (2015). Military culture implications for mental health and nursing care. Online Journal of Issues in Nursing, 20(10). Doi:10.3912/OJIN.Vol20No01Man04

Willis, D.G., Grace, P.J., \& Roy, C. (2008). A central unifying focus for the discipline. Advances in Nursing Science, 31(1), 28-40. doi: 10.1097/01.ANS.0000311534.04059.d9

WVU Office of Research Integrity \& Compliance. (2015). Human research protections program: standard operating procedures. Retrieved at http://oric.research.wvu.edu/files/d/90cce87b-45f8-4ec9-bdad-e5306f39e9b4/wvu-hrppstandard-operating-procedures-3-2016.pdf

Yano, E., Bastian, L., Bean-Mayberry, B., Eisen, S., Frayne, S., Hayes, P., ...Washington, D. (2011). Using research to transform care for women veterans: advancing the research agenda and enhancing research-clinical partnerships. Women's Health Issues. 21(4):S7383. doi: 10.1016/j.whi.2011.04.002

Yano, E., Bastian, L., Frayne, S., Howell, A., Lipson, L., McGlynn, G., ...Fihn, S. (2006). Toward a VA Women's Health Research Agenda: Setting Evidence-based Priorities to Improve the Health and Health Care of Women Veterans. Journal of General Internal Medicine, 21(3), S93-101. doi: 10.1111/j.1525-1497.2006.00381.x 


\section{Appendix A}

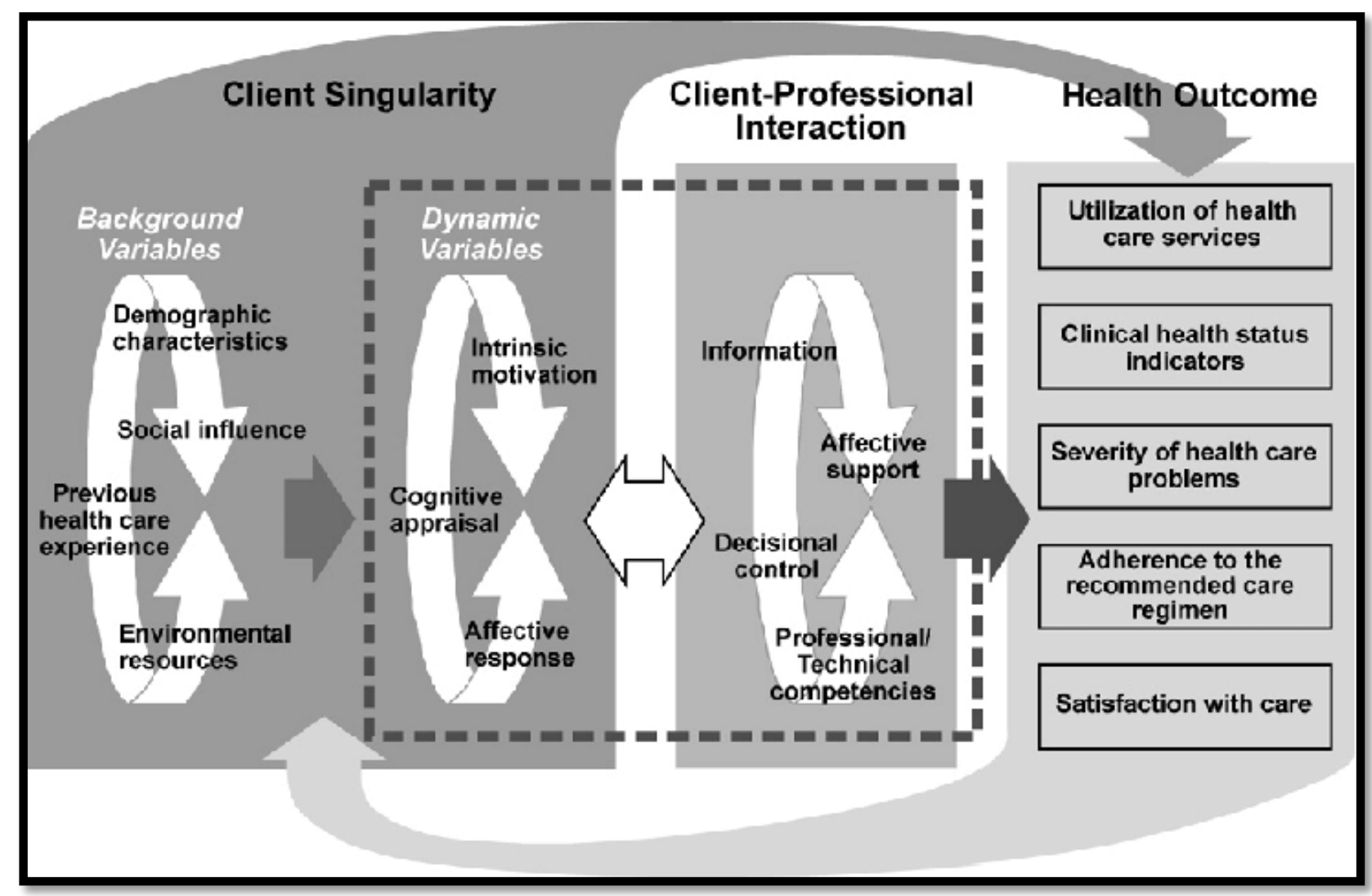

Figure 1. Interaction model of client health behavior

Note. From "A Model of Health Behavior to Guide Studies of Childhood Cancer Survivors," by C. Cox, 2003, Oncology Nursing Forum, 30, E93. Used with permission from the Oncology Nursing Society. 


\section{Appendix B}

Demographic Form

Code \#

\begin{tabular}{|c|c|}
\hline What is your age? & \\
\hline What is your relationship status? & $\begin{array}{ll}\square \text { Single } & \square \text { In a relationship } \\
\square \text { Married } & \square \text { Separated } \\
\square \text { Divorced } & \square \text { Widowed }\end{array}$ \\
\hline What is your race/ethnic background? & $\begin{array}{l}\square \text { American Indian or Alaska Native } \\
\square \text { Asian } \\
\square \text { Black or African American } \\
\square \text { Caucasian } \\
\square \text { Hispanic or Latino } \\
\square \text { Native Hawaiian or Other Pacific Islander }\end{array}$ \\
\hline Do you have health insurance? & $\begin{array}{l}\square \text { yes } \\
\square \text { no } \\
\text { If yes, what type? }\end{array}$ \\
\hline Have you ever sought care from the VHA? & $\begin{array}{l}\square \text { yes } \\
\square \text { no } \\
\text { If yes, what was the result? }\end{array}$ \\
\hline Do you have a service connected disability? & $\begin{array}{l}\square \text { yes } \\
\square \text { no }\end{array}$ \\
\hline $\begin{array}{l}\text { In what branch of the armed forces did/do you } \\
\text { serve? }\end{array}$ & \\
\hline What is your rank? & \\
\hline How many years did you serve in the military? & \\
\hline Were you deployed? & $\begin{array}{l}\square \text { yes } \\
\square \text { no } \\
\text { If yes, where? } \\
\text { How many times? } \\
\text { How long for each deployment? }\end{array}$ \\
\hline What was your role during deployment(s)? & \\
\hline $\begin{array}{l}\text { Were you exposed to harmful agents (oils, fuels, } \\
\text { smoke, toxic gasses, dust, burn pits, insect bites, } \\
\text { etc) during your combat deployment? }\end{array}$ & $\begin{array}{l}\square \text { yes (if yes, please elaborate) } \\
\square \text { no } \\
\square \text { not applicable }\end{array}$ \\
\hline $\begin{array}{l}\text { Did you complete a Post-Deployment Health } \\
\text { Risk Assessment Form (PDHRA) exposure form? }\end{array}$ & $\begin{array}{l}\square \text { yes } \\
\square \text { no If yes, did you report injury or exposure? } \\
\quad \text { Have you had follow up of your injury or exposure? }\end{array}$ \\
\hline
\end{tabular}




\title{
Appendix C
}

\section{Interview Script}

\author{
The purpose of our interview today is to find out more about you and your experience(s) with your healthcare provider(s). \\ Talk to me about how your chose your current healthcare provider. \\ Tell me a bit more about your provider, for instance: is your provider male or female, a generalist or specialist, or an NP, PA, or MD? \\ Tell me what your healthcare provider knows about your veteran status. \\ a. How did they learn this information? \\ Please take a moment and think about a specific health concern that you had that brought you to see your healthcare provider. \\ a. Tell me about your understanding of [insert health concern]. \\ b. Describe what made you bring [insert health concern] to your provider. \\ c. Describe what did you believed or thought was going on with your [insert health concern]. \\ d. Describe how you felt about your [insert health concern].
}

\begin{tabular}{|c|c|}
\hline \multirow[t]{2}{*}{ Affective support } & $\begin{array}{l}\text { Given how you thought and felt about your [insert health concern], describe how your health care provider interacted } \\
\text { with you. }\end{array}$ \\
\hline & $\begin{aligned} \text { Probes: } & \\
\text { 1. } & \text { Tell me how your provider responded to your concerns about your [insert health condition]? } \\
\text { 2. } & \text { Describe how they reacted to your concern. } \\
\text { 3. } & \text { How would you have liked for them to respond? }\end{aligned}$ \\
\hline \multirow{2}{*}{$\begin{array}{l}\text { Information } \\
\text { giving }\end{array}$} & Describe to me the information your provider shared with you about your [insert health concern]. \\
\hline & $\begin{aligned} \text { Probes: } & \\
\text { 1. } & \text { Describe the amount of information provided. } \\
\text { 2. } & \text { Talk to me about your understanding of the information that was provided. } \\
\text { 3. } & \text { Talk to me about your ability to process the information at the time. } \\
\text { 4. } & \text { Describe how you were able to use the information. } \\
\text { 5. } & \text { Describe how you would have liked for your provider to give you information. }\end{aligned}$ \\
\hline \multirow[t]{2}{*}{ Decisional control } & Given your health concern, describe how involved you were in developing your plan of care or treatment. \\
\hline & $\begin{aligned} & \text { Probes } \\
& \text { 1. Describe the level of involvement you wanted to have in your plan of care. } \\
& \text { 2. Describe the level of involvement you think your provider wanted you to have in your care. } \\
& \text { 3. Describe how you feel about how your provider [did or did not] include you in developing a plan of care. } \\
& \text { 4. Describe how you feel about how your provider [did or did not] encourage you in your ability to } \\
& \text { manage/make decisions about your care. } \\
& \text { 5. Describe how the information you received did or did not aide you in making decisions. }\end{aligned}$ \\
\hline \multirow{2}{*}{$\begin{array}{l}\text { Technical- } \\
\text { professional } \\
\text { competence }\end{array}$} & Describe to me the ability of your provider to meet your healthcare needs. \\
\hline & $\begin{array}{cc}\text { Probes: } & \\
\text { 1. } & \text { Describe your confidence in your provider's skill. } \\
\text { a. In treating you as a woman } \\
\text { b. in treating you as a veteran } \\
\text { 2. Describe how the provider met your emotional needs. } \\
\text { 3. Describe how the provider met your physical needs. }\end{array}$ \\
\hline Outcome & Describe to me the outcome of this visit for your health concern. \\
\hline Overall & $\begin{array}{l}\text { We have talked about several things about your experience with your with your health care provider, is there anything } \\
\text { else that you want to share? } \\
\text { Describe to me your overall impression of the care you received by your provider. } \\
\text { Describe to me ways in which you would like your future visits to go? } \\
\text { Consider: We've talked about your experience with your health care provider, such as [insert comments from } \\
\text { participant that are relevant], which aspect is most important to you? }\end{array}$ \\
\hline
\end{tabular}


Appendix D

\section{Categorization Matrix}

\begin{tabular}{|l|l|l|l|}
\hline \multicolumn{1}{|c|}{$\begin{array}{c}\text { Category } \\
\text { (Codes) }\end{array}$} & Specific Descriptors & $\begin{array}{l}\text { Meaning } \\
\text { Units/Condensed } \\
\text { Meaning Units }\end{array}$ & Themes \\
\hline Affective Support & & & \\
\hline $\begin{array}{l}\text { Information } \\
\text { Giving }\end{array}$ & & & \\
\hline $\begin{array}{l}\text { Decisional } \\
\text { Control }\end{array}$ & & & \\
& & & \\
\hline $\begin{array}{l}\text { Professional- } \\
\text { Technical }\end{array}$ & & & \\
\hline
\end{tabular}




\section{Appendix E}

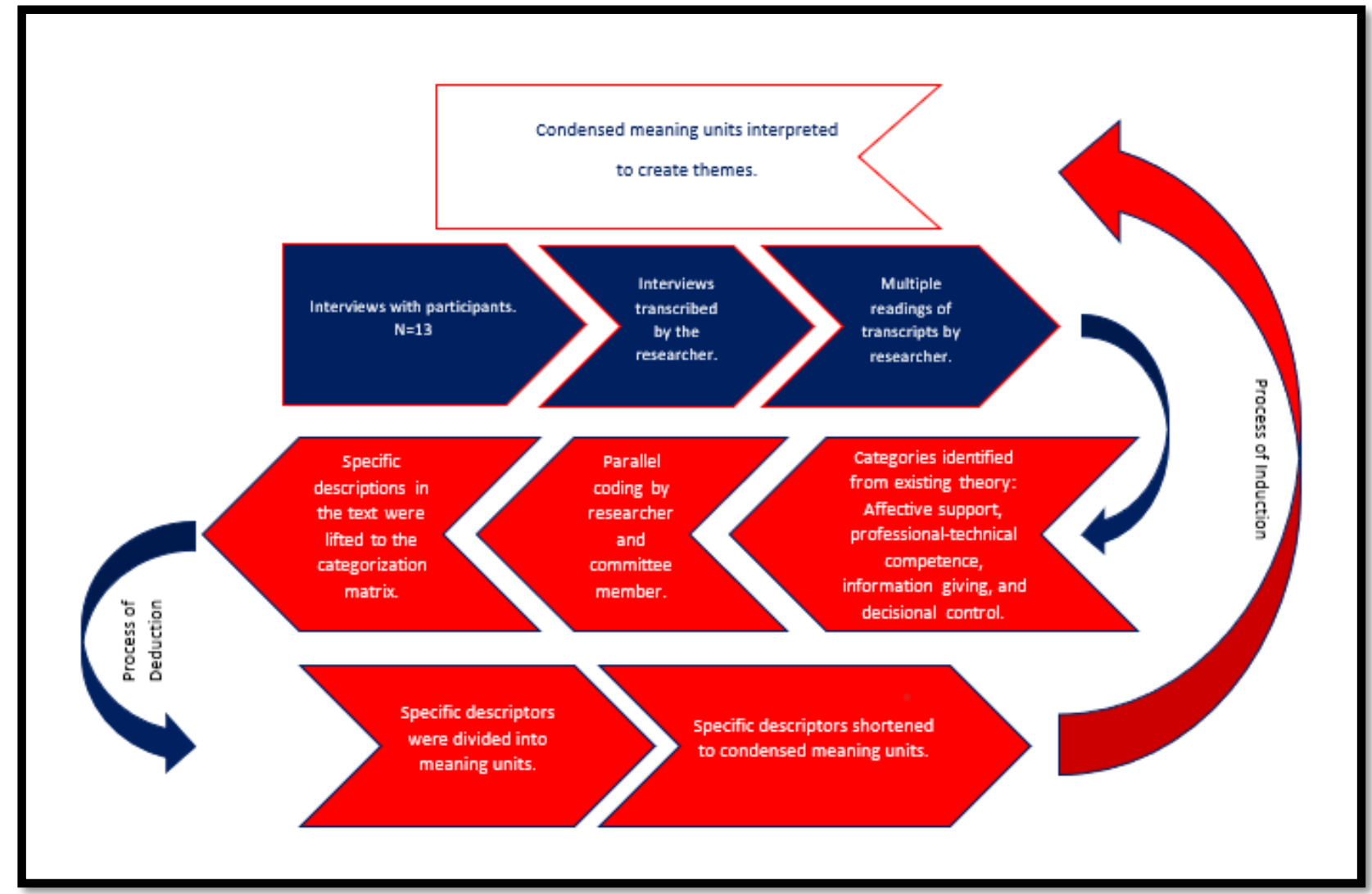

Figure 2. Directed content analysis method 


\section{Appendix F}

\section{Table 1. Themes and Associated Condensed Meaning Units}

\begin{tabular}{|c|c|c|}
\hline Category & "Condensed Meaning Units & Themes \\
\hline $\begin{array}{l}\text { Affective } \\
\text { Support }\end{array}$ & $\begin{array}{l}\text { It is important to know where I come from, about } \\
\text { my service history } \\
\text { Knowing me as a person, not just as my problem } \\
\text { Attentively taking the time to listen and try to } \\
\text { understand how I feel } \\
\text { I can get a response to my concern when I need it, in } \\
\text { a timely manner }\end{array}$ & $\begin{array}{l}\text { Knowing me as a person by hearing my story } \\
\text { and being attentive to my concerns }\end{array}$ \\
\hline $\begin{array}{l}\text { Professional- } \\
\text { technical } \\
\text { Competence }\end{array}$ & $\begin{array}{l}\text { Is it because I am a woman that nobody asked if I } \\
\text { served } \\
\text { They don't think we did the same things as men } \\
\text { I'm not the veteran's wife }\end{array}$ & $\begin{array}{l}\text { Recognizing and acknowledging women are } \\
\text { veterans }\end{array}$ \\
\hline & $\begin{array}{l}\text { They don't understand what I went through } \\
\text { They have little more than a general understanding } \\
\text { of the military } \\
\text { They don't connect a health concern to exposures }\end{array}$ & Comprehending the military experience \\
\hline & $\begin{array}{l}\text { Considering military experience when evaluating } \\
\text { me } \\
\text { Being familiar with my problem and remembering } \\
\text { me increases trust and confidence } \\
\text { Investigating the problem with testing as necessary } \\
\text { Ability to figure out and treat the problem or send } \\
\text { me to someone who can }\end{array}$ & $\begin{array}{l}\text { Being thorough and accepting accountability for } \\
\text { attending to a health concern }\end{array}$ \\
\hline $\begin{array}{l}\text { Information } \\
\text { Giving }\end{array}$ & $\begin{array}{l}\text { Take the time to explain in straight-forward simple } \\
\text { terms } \\
\text { Knowing what is going on empowers decision- } \\
\text { making } \\
\text { Resources are important to help me know what to } \\
\text { expect and what to do }\end{array}$ & $\begin{array}{l}\text { Uncomplicated explanation that facilitates } \\
\text { decision }\end{array}$ \\
\hline $\begin{array}{l}\text { Decisional } \\
\text { Control }\end{array}$ & $\begin{array}{l}\text { It's my body and I want to have a say } \\
\text { Ask me about and help me achieve my goals } \\
\text { We create the plan as a team }\end{array}$ & $\begin{array}{l}\text { Collaborating by seeking input, providing } \\
\text { options, and supporting deons }\end{array}$ \\
\hline
\end{tabular}

